(c) 2008 Elsevier Ltd All rights reserved.

\title{
How momentum advection schemes influence current-topography interactions at eddy permitting resolution
}

\author{
Julien Le Sommer ${ }^{a,{ }^{*}}$, Thierry Penduff ${ }^{a}$, Sébastien Theetten ${ }^{b}$, Gurvan Madec ${ }^{c}$ and Bernard \\ Barnier $^{a}$
}

\author{
${ }^{a}$ CNRS, UJF, INPG, UMR5519, F-38041 Grenoble, France \\ ${ }^{\mathrm{b}}$ CNRS, IFREMER, UBO, UMR6523, F-29280 Plouzané, France \\ ${ }^{\mathrm{c}}$ CNRS, IPSL, UPMC, UMR7159, F-75252 Paris, France
}

*: Corresponding author : Julien Le Sommer, Tel.: +33 4768250 65; fax: +33 4768252 71, email address : lesommer@hmg.inpg.fr

\begin{abstract}
:
Recent studies have shown that the use of an enstrophy-and-energy-conserving momentum advection scheme substantially reduces widespread biases of mean currents in the global $1 / 4^{\circ}$ DRAKKAR model. This paper investigates the origin of these improvements. A series of sensitivity simulations with different momentum advection schemes is performed with the North Atlantic 1/4 ${ }^{\circ}$ DRAKKAR model. Three second order momentum advection schemes conserving, respectively, enstrophy (ens), energy (efx) and both quantities (een) are tested and their impact on the model solution are compared.

The mean kinetic energy vertical profile is found to change up to $10 \%$ depending on the chosen scheme. This sensitivity is maximum in bottom layers. The analysis of the vorticity tendency due to horizontal momentum advection reveals that the three schemes differ mostly in bottom layers as well. The average magnitude of this term is enhanced with the efx scheme and reduced with the een scheme. These differences are found to be consistent with the instantaneous tendency of each scheme.

In addition, we show that the differences between the schemes are related to the grid-scale irregularity of the velocity field. Both the grid scale irregularity and the differences between the schemes are found to be enhanced in bottom layers. We conclude that the model solution depends crucially on the ability of the momentum advection scheme to handle under-resolved flows close to the bottom topography. This work emphasizes the critical influence of topography in eddy-active regions on mean circulation features such as the position of the North-Atlantic current or the Gulf Stream separation.
\end{abstract}

Keywords: Momentum advection schemes; Topography; z-level models 


\section{Introduction}

Models intercomparison and sensitivity studies have proved to be fruitful approaches both to improve ocean climate models and to help understanding ocean dynamics. Regarding the North-Atlantic basin, such experiments include, among others, the DYNAMO project (Willebrand et al., 2001), and the DAMEE-NAB project (Chassignet et al., 2000). These experiments have notably illustrated the sensitivity of basin scale circulation with respect to vertical coordinate formulation. More generally, over the past two decades, an intense focus has been put on improving the treatment of vertical physics (Large et al., 1994) and the topographic constraint on deep overflows (Beckmann and Döscher, 1997; Pacanowski and Gnanadesikan, 1998). Likewise, considerable achievements have been made in the representation of tracer transport processes by improving tracer advection schemes (see e.g. Gerdes et al., 1991; Webb et al., 1998) and introducing parameterizations of lateral mixing and eddy stirring (Gent and McWilliams, 1990; Gent et al., 1995). The interested reader would find a comprehensive description of the recent developments in ocean climate modelling with detailed references in the review paper by Griffies et al. (2000). Still, in the same period, the dynamical cores, i.e. the momentum equations, of our models were relatively less discussed.

Besides the fundamental improvements mentioned above, the evolution of computer resources has a strong influence on the solutions of model simulations. Indeed, given the increase in resolution, the decrease in the amount of dissipation required to insure stability yields significant changes in the flow regimes actually simulated. Currents and their vertical structures are reproduced more accurately (see e.g. Penduff et al., 2005) so that ocean simulations 
now provide more information than only water mass properties. In the same way, the nonlinearity is enhanced, i.e. the flows exhibit higher Rossby and Reynolds numbers. This, in turn, supports more intense vortex interactions and current-topography interactions. An illustration of the effect of currenttopography interactions on the mean flows is given by Merryfield and Scott (2006) who have recently discussed the paramount effect of model resolution on the orientation of deep currents in a global ocean model. However, most eddyadmitting, z-level model simulations exhibit discrepancies in the circulation patterns (path of the Gulf stream, North Atlantic Current system, path and width of the Deep Western Boundary Current), in the vertical mean kinetic energy (MKE) and eddy kinetic energy (EKE) profiles (see e.g. Willebrand et al., 2001; Penduff et al., 2001).

Recently, in the context of the DRAKKAR project ${ }^{1}$, Barnier et al. (2006) and Penduff et al. (2007) have shown that the combined use of a partial step topography (Adcroft et al., 1997) and a momentum advection scheme conserving total enstrophy and kinetic energy yields substantial improvement of mean circulation patterns in their $1 / 4^{\circ}$ global ocean model. In particular, noticeable biases in the North Atlantic (including the large eddy north of Cape Hatteras, the absence of strong recirculation north and south of the Gulf Stream and the absence of North-West Corner) were significantly reduced. Likewise, these modifications were shown to reduce the overestimated vertical dependence of the MKE and EKE vertical profiles. Extending these results, we focus in this paper on the choice of the momentum advection scheme, the effect of partial step topography being investigated elsewhere (Penduff et al., 2007). More precisely, the purpose of the present study is twofold : $(i)$ evaluating

$\overline{1 \text { http://www.ifremer.fr/lpo/drakkar/ }}$ 
the degree of sensitivity of eddy-admitting, z-coordinate ocean simulations to the choice of a momentum advection scheme and (ii) investigating the dynamical origin of this sensitivity. To that purpose, sensitivity experiments were carried out with a North-Atlantic model configuration extracted from the $1 / 4^{\circ}$ global DRAKKAR model (Barnier et al., 2006). Along each model solution, the behavior of the momentum advection scheme is diagnosed. A method is proposed in order to pinpoint the origin of the mismatch between momentum advection terms as calculated with different schemes.

The paper is organized as follows. We briefly review in section 2 the discretization strategies currently used for momentum advection terms in most OGCMs and describe the schemes used throughout this study. Section 3 describes the North Atlantic model configuration and our methodology. The behavior of the schemes and their impact on the circulation is diagnosed in section 4 . In section 5, we discuss a possible mechanism responsible for the sensitivity regarding momentum advection schemes. The results are discussed and put into perspective in section 6 .

\section{On momentum advection schemes}

\subsection{General formulation}

We focus on the discretization of the momentum advection term in the horizontal momentum equation of the primitive equations. For reasons which will appear more obvious hereafter, it is customary to consider this term together with the term due to Coriolis force. We therefore examine the collected term 
$\mathcal{T}_{a c}$, written in an Cartesian frame in the form

$$
\mathcal{T}_{a c}=\mathbf{v} \cdot \nabla \mathbf{u}+f \mathbf{k} \times \mathbf{u}
$$

where $\mathbf{v}=(u, v, w)^{T}$ is the full three-dimensional velocity vector and $\mathbf{u}=$ $(u, v, 0)^{T}$ designates its horizontal component. Superscripts ${ }^{T}$ indicate matrix transposition.

The term $\mathcal{T}_{a c}$ is usually discretized starting from one of the two following equivalent formulations. One can write $\mathcal{T}_{a c}$ following the vector-invariant formulation as

$$
\mathcal{T}_{a c}=\frac{1}{2} \nabla_{h}\left(\mathbf{u}^{2}\right)+w \partial_{z} \mathbf{u}+(f+\zeta) \mathbf{k} \times \mathbf{u}
$$

where $\zeta$ denotes the vertical component of the relative vorticity, i.e. $\zeta=$ $\left(\partial_{x} v-\partial_{y} u\right)$, and $\nabla_{h}=\left(\partial_{x}, \partial_{y}, 0\right)^{T}$ is the horizontal component of the gradient operator. This form is independent on the coordinate frame. In this form, the relative vorticity term has the same form as the term due to Coriolis force. Both terms are then usually treated with the same numerical approach.

An alternate approach uses the continuity equation to obtain the flux form

$$
\mathcal{T}_{a c}=\nabla \cdot\left(\mathbf{v}^{T} \otimes \mathbf{u}\right)+f \mathbf{k} \times \mathbf{u}
$$

where $\otimes$ designates the Kronecker product. Like form (1), the latter form (3) depends on the coordinate frame. More precisely, metric terms should be added to these forms when written in a curvilinear coordinate frame.

Although both formulations (2) and (3) are equivalent in the continuous limit, they yield two distinct classes of numerical schemes with distinct properties. Indeed, discretizing the vector invariant form (2) yields intrinsic constrains on the vorticity budgets whereas discretizing the flux form (3) more naturally 
constraints energy budgets. Whatever their vertical coordinate and horizontal grids, all the OGCMs are based on one of the forms (2) or (3). For instance, both formulations are available in NEMO (Madec, 2006) and the MIT-GCM (Adcroft et al., 2004). MOM (Pacanowski and Griffies, 1999) is implemented with form (3). Likewise, isopycnal models are also based on this formalism: HIM (Hallberg, 1997) uses form (2) and MICOM (Bleck et al., 1992) uses form (3), both discretized on C-grids.

\subsection{Description of the schemes}

Having noted in the introduction the high sensitivity of ocean models to their Coriolis-momentum advection scheme, our purpose is not to design a new Coriolis-momentum advection scheme but rather to pinpoint the origin of such sensitivity. To that purpose, we compare three second order Coriolismomentum advection schemes among the most classically used for C-grid ocean models. The arrangement of the variables on a C-grid is summarized in Table 1.

[Table 1 about here.]

(a) The first scheme is based on form (2) and is discussed by Sadourny (1975). When applied to the shallow water equations, it guarantees the conservation of the basin-integrated potential enstrophy (i.e. the mean square potential vorticity). For the primitive equations, an additional term associated with vertical advection appears, namely $w \partial_{z} \mathbf{u}$. This vertical advection term and the horizontal gradient of horizontal kinetic energy term are discretized with second order finite differences following Madec et al. 
(1998). As noted by these authors, the latter two terms cannot affect the total kinetic energy budget.

Hereafter, the combined scheme is referred to as scheme ens.

(b) The second scheme is also based on form (2). It was derived by Sadourny and subsequently tested by the European Center for Medium Range Forecasts (Burridge and Haseler, 1977). Its conservation properties are discussed by Arakawa and Lamb (1981). For the shallow water equations, it conserves both basin-integrated potential enstrophy (for non-divergent flows) and kinetic energy (for general flows). The horizontal gradient of horizontal kinetic energy and the vertical advection terms are treated as for the ens scheme. The scheme is referred to as the een scheme.

(c) The third scheme is the most simple second order scheme based on form (3) which guarantees the conservation of total (i.e. three-dimensional) kinetic energy. It is based on the general method proposed by Bryan (1966). In this sense, it can be considered as a C-grid equivalent of the B-grid scheme used in the early versions of GFDL model and described by Bryan (1969). In addition, the Coriolis term is discretized following the stencil of the een scheme, with no consequence on the kinetic energy budget. This scheme is referred to as the efx scheme.

It is worth noting that the use of form (2) allows one to impose separate integral constraints on the total vorticity term on the one hand, and on the vertical advection and gradient of kinetic energy term on the other hand. On the contrary, the use of form (3) yields integral constraints only on $\mathcal{T}_{a c}$.

Setting apart the computation of the Coriolis term, the ef $x$ scheme involves 
the computation of fluxes between adjacent cells. Indeed, remembering that in a Cartesian frame,

$$
\nabla \cdot\left(\mathbf{v}^{T} \otimes \mathbf{u}\right)=\left\{\begin{array}{l}
\partial_{x}\left(u^{2}\right)+\partial_{y}(u v)+\partial_{z}(u w) \\
\partial_{x}(v u)+\partial_{y}\left(v^{2}\right)+\partial_{z}(v w)
\end{array},(4)\right.
$$

the update of, say, the $u$-component of the momentum equations at point $(i, j+1 / 2, k)$ is obtained by computing derivatives of three quadratic quantities in the relevant spatial directions. As with any standard finite volume approach (LeVeque, 1992), the derivatives are computed as flux differences across surfaces normal to the axis being considered. In addition, following Bryan (1966), the quadratic fluxes are defined in the volumes adjacent to the surface with interpolation formulae which avoid nonlinear instability. For the $u$-component of the momentum equations and on a regular grid with constant grid spacing, this yields the interpolation formulae

$$
\begin{array}{ll}
{\left[u^{2}\right]_{i+\frac{1}{2}, j+\frac{1}{2}, k}} & =\left(\bar{u}^{i+\frac{1}{2}, j+\frac{1}{2}, k}\right)^{2} \\
{[u v]_{i, j, k}} & =\left(\bar{u}^{i, j, k}\right)\left(\bar{v}^{i, j, k}\right) \\
{[u w]_{i, j+\frac{1}{2}, k+\frac{1}{2}}} & =\left(\bar{v}^{i, j+\frac{1}{2}, k+\frac{1}{2}}\right)\left(\bar{w}^{i, j+\frac{1}{2}, k+\frac{1}{2}}\right)
\end{array}
$$

with overbars indicating spatial averages over a unitary volume centered on the referred indices. Then, the flux differences are computed at point $(i, j+1 / 2, k)$ by subtracting the neighboring values along the axis of the derivative, so that, e.g.

$$
\left[\partial_{y}(u v)\right]_{i, j+\frac{1}{2}, k}=\left([u v]_{i, j+1, k}-[u v]_{i, j, k}\right) / \Delta y
$$

where $\Delta y$ is the meridional grid spacing. As mentioned above, we treat the remaining Coriolis term as with the een scheme. 
The differences between the spatial stencils of the ens scheme and the een scheme are schematically illustrated in Fig. 1.

[Fig. 1 about here.]

The two schemes differ only in the treatment of the total vorticity term, $(f+$ $\zeta) \mathbf{k} \times \mathbf{u}$. For example, for the ens scheme, the term $(f+\zeta) v$, is estimated at point $(i, j+1 / 2, k)$ following

$$
[(f+\zeta) v]_{i, j+\frac{1}{2}, k}=\left(\bar{v}^{i, j+\frac{1}{2}, k}\right)\left(\overline{f+\zeta}^{i, j+\frac{1}{2}, k}\right) .
$$

On the other hand, the spatial stencil of the een scheme involves the computation of four triads, namely,

$$
\begin{aligned}
& \Lambda\urcorner_{i, j+\frac{1}{2}}=\left([f+\zeta]_{i-1, j+1}+[f+\zeta]_{i, j+1}+[f+\zeta]_{i, j}\right) / 3 v_{i-1 / 2, j+1} \\
& \Lambda\rfloor_{i, j+\frac{1}{2}}=\left([f+\zeta]_{i-1, j}+[f+\zeta]_{i, j}+[f+\zeta]_{i, j+1}\right) / 3 v_{i-1 / 2, j} \\
& \Lambda r_{i, j+\frac{1}{2}}=\left([f+\zeta]_{i+1, j+1}+[f+\zeta]_{i, j+1}+[f+\zeta]_{i, j}\right) / 3 v_{i+1 / 2, j+1} \\
& \Lambda\left\llcorner{ }_{i, j+\frac{1}{2}}=\left([f+\zeta]_{i+1, j}+[f+\zeta]_{i, j}+[f+\zeta]_{i, j+1}\right) / 3 v_{i+1 / 2, j}\right.
\end{aligned}
$$

where the subscript $k$ is omitted for simplicity. Then, the triads are combined within the stencil

$$
\begin{aligned}
& {[(f+\zeta) v]_{i, j+\frac{1}{2}}=} \\
& \left.\quad[\Lambda\urcorner_{i, j+1 / 2}+\Lambda\right\lrcorner_{i, j+1 / 2}+\Lambda\left\ulcorner_{i, j+1 / 2}+\Lambda\llcorner i, j+1 / 2] / 4 .\right.
\end{aligned}
$$

It is interesting to note that this triad formulation is very similar to the stencil proposed by Griffies et al. (1998) for the treatment of iso-neutral tracer diffusion in z-level models. The triad formulation of Griffies et al. (1998) guarantees the decrease of tracer variance whereas, in our case, it enforces energy 
conservation.

It is worth noting that the three schemes also slightly differ in the implementation of the lateral boundary conditions. For the three schemes, a free-slip boundary condition is used so that the normal component of velocity and the relative vorticity are explicitely set to zero at the boundaries. Moreover, for the een scheme (and for the Coriolis term of the efx scheme), the planetary vorticity $f$ is weighted by the average layer thickness at the four neighboring T-points. This choice, which does not change the integral properties of the schemes, is expected to give smoother solutions near the boundaries with the een scheme by effectively shaving the cell depth at $f$-points. We emphasize that the choice of an appropriate boundary condition for planetary vorticity on a C-grid is a longstanding issue (see e.g. Jamart and Ozer, 1986) still under debate.

In addition to the integral properties mentioned above, Arakawa and Lamb (1981) observed that, for the inviscid shallow water equations, the Lagrangian conservation of potential vorticity was more accurately preserved by the een scheme than by the ens scheme. Recall that the integral properties of the schemes ens and een are guaranteed for barotropic flows only since they have been derived within the shallow water framework. It is also worth noting that the schemes considered are not unique in having these particular integral conservation properties. An interested reader would find a comprehensive presentation of the schemes available in the shallow water framework and their derivation in Salmon (2004). Going back to the three dimensional case, the quasi two-dimensional dynamics of oceanic flows suggest that twodimensional conservation properties might improve the simulated flows. Still, the constraint on energy budgets and energy spectra in the three-dimensional 
case is unknown. Although there is not an extensive literature on the topic, some papers have pointed out the sensitivity of three-dimensional ocean simulations to momentum advection scheme, most of them in a B-grid framework (see e.g. Webb et al., 1998; Ishizaki and Motoi, 1999). But, to our knowledge, there is no detailed discussion of the effect of the schemes een,ens and efx on large scale three-dimensional ocean simulations nor any disclosure of the mechanism involved in this sensitivity. This is one of the purposes of the following sections.

\section{Numerical configuration, simulations and methodology}

\subsection{Description of the three DRAKKAR-NATL025 runs}

The North-Atlantic model used herein is based on NEMO which combines the free-surface, z-coordinate, primitive equation code OPA9 (Madec et al., 1998; Madec, 2006) and the multi-layered sea-ice code LIM2 (Fichefet and Morales Maqueda, 1997). The North Atlantic configuration has been developed in the context of the DRAKKAR project. It is build up as a regional extraction of the global DRAKKAR-ORCA-R025 configuration (Barnier et al., 2006). Herein, we only provide a short overview of the model configuration and forcing fields. The interested reader can refer to Barnier et al. (2006) for a thorough description.

The horizontal mesh is a regular Mercator grid with $1 / 4^{\circ}$ resolution at the equator which covers the Atlantic Ocean and the Nordic Seas from latitude $20^{\circ} \mathrm{S}$ to $80^{\circ} \mathrm{N}$. The vertical grid has 46 geopotential levels with a grid spacing ranging from $6 \mathrm{~m}$ at the surface to $250 \mathrm{~m}$ for the lowermost level. The bottom 
topography is represented by full cells where depths result from an interpolation of ETOPO2 dataset as shown in Fig. 2. Buffer zones are defined at the northern boundary, at the southern boundary and in the eastern Mediterranean sea. In this respect, the model configuration repeats the methodology adopted by the DYNAMO project (Willebrand et al., 2001) but with a higher resolution and a computational domain extending further north.

[Fig. 2 about here.]

Lateral parameterizations include isopycnal Laplacian diffusion of tracers $\left(\kappa_{\rho}\right)$, biharmonic diffusion of momentum $\left(\kappa_{\mu}\right)$. Moreover, $\kappa_{\rho}$ and $\kappa_{\mu}$ decrease from the equator (where $\kappa_{\rho}=300 \mathrm{~m}^{2} . \mathrm{s}^{-1}$ and $\kappa_{\mu}=-1.510^{11} \mathrm{~m}^{4} \cdot \mathrm{s}^{-1}$ ) toward the pole at the first and third power of the grid step respectively. The parameterization of vertical physics is achieved with a second order closure model plus an enhanced vertical mixing of tracers and momentum in case of static instability. In addition, a quadratic bottom friction and free slip lateral boundary conditions are used.

As in Barnier et al. (2006), initial conditions for temperature and salinity are derived from the Levitus et al. (1998) dataset except for the high latitudes where the PHC2.1 (Steele et al., 2001) climatology has been chosen and the Mediterranean Sea which was initialized with the Medatlas (Jourdan et al., 1998) climatology. Monthly means of the same datasets are used to specify the relaxed condition in the buffer zones. At the surface, the ocean-sea ice model is forced with surface momentum flux, provided in the form of a wind stress vector, and surface heat and fresh water fluxes calculated using empirical bulk parameterizations (see Barnier et al., 2006, for details). All the input fields are combined in a climatological year applied as a cycling forcing. The wind stress 
vector we used is a daily mean wind stress vector obtained from a blend of ERS scatterometer data (CERSAT 2002) and NCEP/NCAR reanalysis (Kalnay et al., 1996). The set of atmospheric variables used in the bulk parameterizations is described in Barnier et al. (2006). In particular, it includes CMAP (Xie and Arkin, 1997) climatological monthly mean precipitation.

For this study, three 11-year simulations have been performed : one with the scheme ens (hereafter referred to as run ENS), one with the scheme een (run EEN) and one with the scheme efx (run EFX). Run EEN uses the same numerics as Barnier et al. (2006)'s run G03 whereas run ENS is equivalent to their run G04. Except for the Coriolis-momentum advection scheme, the whole model configuration, including the initial fields and the forcing fields, are identical in the three simulations.

The model solutions compare well to other North-Atlantic model simulations at comparable resolution for what concerns the mean meridional circulation (not shown) and the large-scale horizontal circulation.

\subsection{Mean circulation sensitivity to momentum advection schemes}

Depending on the chosen scheme, we expect the mean flows to differ significantly in regions which dynamics is known to be influenced by flow-topography interactions. In this respect, the Gulf Stream region, which has been thoroughly discussed in the literature, is certainly a good test case. The sensitivity of the mean surface circulation in this region is illustrated in Fig. 3, showing the mean sea surface height in run EEN, ENS and EFX. As expected, the mean flows differ significantly in this region. More precisely, the sensitivity 
diagnosed in our DRAKKAR-NATL025 configuration is consistent with the conclusions of Barnier et al. (2006) (see their Fig. 7). Indeed, the three runs differ mostly in the representation of critical circulation patterns : the erroneous presence of a large eddy north of Cape Hatteras, the abrupt steering of the mean current to the north at $45^{\circ} \mathrm{W}$ near the Mann eddy and then to the east near $51^{\circ} \mathrm{N}$ around the North-West Corner. Run EEN with the een scheme is the closest to the climatology proposed by Niiler et al. (2003). Run ENS and EFX exhibit biases, namely a large eddy north of Cape Hatteras, no abrupt steering of the mean current to the north and no well defined Mann eddy and North West Corner. In addition, run EFX displays several blocked eddies which are not consistent with observations, e.g. south of Cape Hatteras (see Fig. 3) and in the Gulf of Mexico (not shown). Note that such a blocking of the Loop Current in the Gulf of Mexico is a common bias which was reported in several other model studies (see e.g. Smith et al., 2000; Candela et al., 2003).

[Fig. 3 about here.]

As illustrated by the mean kinetic energy vertical profiles in Fig. 5, the vertical structure of the flow is also very sensitive to the Coriolis-momentum advection scheme. In Fig. 5, the vertical profiles are computed on surfaces of constant level above the bottom topography, as described in Fig. 4. This convention has been chosen to emphasize the critical influence of the distance to the bottom topography on the vertical profiles. The resulting vertical profiles happen to be smoother than z-level averaged vertical profiles thus yielding a clearer picture of the sensitivity to numerical schemes and to the distance to the bottom boundary. Fig. 5 shows that, compared to the ens scheme, the een scheme (efx) tend to strengthen (slow down) mean currents in the deep ocean and 
to slow down (strengthen) mean currents in the upper layers. In this sense, the een scheme reduces the vertical dependence of the MKE whereas the ef $x$ scheme increases the vertical dependence of the MKE ${ }^{2}$.

[Fig. 4 about here.]

[Fig. 5 about here.]

It is notable that the difference from run to run is enhanced close to the topography. These results are consistent with the results of Penduff et al. (2007), who have also diagnosed the effect of the schemes een and ens in a series of global $1 / 4^{\circ}$ simulations. Their study has shown that the choice of the Coriolisadvection scheme could affect both the mean and eddy ciculation and that this sensitivity was enhanced close to the topography in the bottom layers. It is therefore sound to seek the origin of the sensitivity of the mean circulation (diagnosed in Fig. 3) in bottom layers. This is why, for all the vertical profiles presented hereafter we restrict the analysis to depth greater than $1000 \mathrm{~m}$. The averaging method defined in Fig. 4 then allows one to investigate the dependence to the distance to topography in bottom layers without mixing information from the upper layers.

\subsection{Diagnostic approach}

In order to understand the dynamical origin of the sensitivity to the Coriolismomentum advection scheme, we set apart the integral conservation properties of the schemes, which give little indication in the three-dimensional case, and

$\overline{2}$ For concision, the ens scheme will be used hereafter as a reference scheme to which schemes een and $e f x$ are compared. 
focus on the actual behavior of the schemes. Therefore, we choose to examine the differences of the Coriolis-momentum advection terms along the model solution. Yet, the foreseeable discrepancies between the terms as computed along each model solution can be due to two distinct effects. Indeed, one has to distinguish the instantaneous mismatch between the schemes for a given velocity field from the differences due to the cumulative effect of the scheme on the flow (i.e. on the velocity field). Hereafter, we use the terms instantaneous tendency and cumulative change to designate the two effects.

[Fig. 6 about here.]

In order to separate the instantaneous and the cumulative effects on the behavior of the Coriolis-momentum advection schemes, we use the diagnostic approach illustrated in Fig. 6. Along each model solution, the Coriolismomentum advection terms as computed by the three schemes have been stored. Note that only one of the methods is actually used to update the model state variables. By examining the differences between the three tendencies, we will possibly localize where the Coriolis-momentum advection schemes tend to spread the model solutions.

\section{Momentum advection schemes and vorticity dynamics}

We focus on the contribution of $\mathcal{T}_{a c}$ to the vorticity tendency. With $1 / 4^{\circ}$ horizontal resolution, scaling arguments suggest that the main difference from scheme to scheme should come from the horizontal advection part of $\mathcal{T}_{a c}$, i.e. $\mathbf{u} \cdot \nabla \mathbf{u}$. Indeed, even though relative vorticity is smaller than planetary vorticity in the simulations (with usual Rossby numbers $R_{0}<0.1$ ), the grid- 
scale variations of relative vorticity largely exceeds the planetary $\beta$ term. The treatment of the Coriolis term is critical at lower resolutions (see e.g. Nechaev and Yaremchuk, 2004), due to the significant variation of Coriolis parameter at the grid scale. But, in the simulations presented in this paper, the $\beta$-effect is dominated by the differences due to $\mathbf{u} \cdot \nabla \mathbf{u}$. Therefore, we restrict our analysis to $\mathcal{T}_{\zeta}$, the vorticity tendency due to horizontal advection. For continuous fields, it is equal to the divergence of the horizontal fluxes of relative vorticity,

$$
\mathcal{T}_{\zeta}=[\nabla \times(-\mathbf{u} \cdot \nabla \mathbf{u})] \cdot \mathbf{z}=\nabla_{h} \cdot(\zeta \mathbf{u})
$$

In what follows, $\mathcal{T}_{\zeta}$ has been computed with the model second order curl operator. We stress that the quantity $\mathcal{T}_{\zeta}$ is a three-dimensional variable which varies in time. Fig. 7 shows a snapshot of the surface $\mathcal{T}_{\zeta}$ in the Gulf stream region in run EEN. As can be expected, $\mathcal{T}_{\zeta}$ varies at scales typical of oceanic meso-scale vortices. Since meso-scale vortices are only marginally resolved at $1 / 4^{\circ}$ horizontal resolution, $\mathcal{T}_{\zeta}$ exhibits a significant grid-scale variability. The lower panel of Fig. 7 presents the surface $\mathcal{T}_{\zeta}$ along the black line of the upper panel as computed with the three different schemes along the run EEN. The agreement between the schemes is correct except for some very local values. More precisely, it appears that the terms mostly differ in their intensity rather than in their patterns. It is striking that local values can change up to $30 \%$ depending on the scheme. In particular, the scheme ef $x$ tends to produce more intense peak values whereas the een scheme tends to produce reduced peak values.

[Fig. 7 about here.]

In order to pinpoint the origin of long-term modifications of the mean flow field, we seek for systematic differences between the schemes. We therefore 
focus our analysis on the mean divergence defined below,

$$
T\left(k_{u p}\right)=\left\langle\left|\overline{\mathcal{T}}_{\zeta}^{t}\right|\right\rangle_{k_{u p}=c s t}=\left\langle\left|{\overline{\nabla_{h} \cdot(\zeta \mathbf{u})}}^{t}\right|\right\rangle_{k_{u p}=c s t}
$$

where $\digamma^{t}$ is the time average and $\langle\cdot\rangle_{k_{u p}=c s t}$ designates the spatial average over levels of constant $k_{u p}$. The time average is defined over year 11 and the spatial average over the whole computational domain. The definition of $k_{u p}$ at $f$-points is given in Fig. $4 . T\left(k_{u p}\right)$ is the average magnitude of the vorticity tendency due to horizontal advection at level $k_{u p}$ above the topography. Note that various definitions of $k_{u p}$ regarding the treatment of bottom boundary points have been tested without changing the main conclusions presented hereafter.

Fig. 8 shows the vertical profile $T\left(k_{u p}\right)$ as computed along each model solution. We first note that, in the three simulations, the vorticity tendency due to horizontal advection increases close to the bottom topography. This is probably what is to be expected in regions where vorticity gradients are relatively bigger, namely near the boundaries. Second, it appears that $T\left(k_{u p}\right)$ is systematically intensified along the run EFX and reduced along the run EEN. Third, these systematic differences are maximum close to the bottom topography.

[Fig. 8 about here.]

At this point, one may ask whether these systematic discrepancies between the terms are due to the different circulations of each run or to the instantaneous mismatch between the schemes. That is, we should distinguish the instantaneous tendency of the schemes and the effect of the cumulative change. Fig. 9 presents the terms $T\left(k_{u p}\right)$ as computed along the same model solution (EFX). Similar results can be obtained along EEN and ENS solutions (not shown). 
Although the discrepancies shown in Fig. 9 appear to be slightly bigger than in Fig. 8, the similarity with Fig. 8 is striking. More precisely, it indicates that, on average, (i) the efx scheme produces more intense $T\left(k_{u p}\right)$, (ii) the een scheme produce less intense $T\left(k_{u p}\right)$.

[Fig. 9 about here.]

It is worth noting that the intensification with the efx scheme and the reduction with the een scheme are also observed on instantaneous spatially averaged $\left|\mathcal{T}_{\zeta}\right|$ profiles (not shown). As a conclusion, the differences in $T\left(k_{u p}\right)$ between run EEN, ENS and EFX are consistent with the instantaneous tendency of each scheme.

\section{Impact of the regularity of the velocity field}

\subsection{Scheme consistency and regularity of the input velocity field}

We have shown that the terms differ mostly in bottom layers. Therefore, some flow property is most certainly altered in these layers whatever the scheme used to perform the simulation. In order to assess what flow property is responsible for the differences between the schemes, it is profitable to recall that the three schemes are consistent. That is, considering a continuous velocity field sampled at some grid scale $h>0$, the three schemes are known to converge toward the same value with decreasing $h \rightarrow 0$. For a fairly general class of velocity fields, decreasing $h \rightarrow 0$ implies that the remainder of a Taylor series expansion of

$\mathbf{u}$ goes to zero. Reversing the argument, an equivalent statement tells that for a given grid scale $h>0$, the mismatch between the schemes decreases with 
increasing regularity of the input velocity field. More precisely, for a given velocity field $\mathbf{u}$, consider its first order Taylor series expansion at point $\mathbf{x}$ in the vicinity of point $\mathbf{x}_{\mathbf{0}}$

$$
\mathbf{u}=\mathbf{u}_{\mid \mathbf{x}^{\prime}=\mathbf{x}_{\mathbf{0}}}+\left(\mathbf{x}-\mathbf{x}_{\mathbf{0}}\right) \cdot \nabla \mathbf{u}_{\mid \mathbf{x}^{\prime}=\mathrm{x}_{\mathbf{0}}}+\mathcal{O}\left(\left\|\mathbf{x}-\mathbf{x}_{\mathbf{0}}\right\|^{2}\right)
$$

For simplicity, we only discuss hereafter the effect of horizontal discretization, so $\mathbf{u}$ denotes the horizontal velocity field and $\mathbf{x}$ is a two-dimensional spatial variable $\mathbf{x}=(x, y)$. Moreover, the Taylor series expansion is restricted to first order because our schemes only involve second order discretizations and therefore do not provide information on higher order quantities. Now, consider a grid with a given grid scale $h$ and let $U$ denote the typical order of magnitude of $\mathbf{u}, \delta U$ a typical scale of the first order term in the Taylor series at the grid scale (e.g. $h\|\nabla \mathbf{u}\|$ ) and $\delta^{2} U$ the typical scale of the remaining term in the Taylor expansion (11). At the grid scale $h$, such a velocity field $\mathbf{u}$ with typical scales $U, \delta U$ and $\delta^{2} U$ can be characterized by two non-dimensional parameters, say

$$
R_{U}=\delta U / U \quad \text { and } \quad R_{\Delta U}=\delta^{2} U / \delta U
$$

The first parameter $R_{U}$ is the relative order of magnitude of the velocity gradients at the grid scale. In this sense, $R_{U}$ is an indicator of the flow regime. The second parameter $R_{\Delta U}$ is the relative order of magnitude of the variations of the velocity gradients at the grid scale. For velocity fields obtained with second order schemes, it indicates how noisy are the velocity gradients at the grid scale. With these notations, the consistency of the second order schemes een, ens and efx implies that, for fixed $R_{U}$, the values of $\mathcal{T}_{a c}$ as calculated by the three schemes will converge as $R_{\Delta U} \rightarrow 0$. 
In order to state what is the dominant effect responsible for the variation of the ratio of momentum advection terms (e.g. $\left.\left|\mathcal{T}_{\zeta}^{\text {een }}\right| /\left|\mathcal{T}_{\zeta}^{\text {ens }}\right|\right)$ in the simulations, we would like to describe completely the variations of this ratio with respect to $R_{U}$ and $R_{\Delta U}$. First, the consistency argument mentioned above states that the ratios tend to 1 as $R_{\Delta U} \rightarrow 0$. Second, for schemes een and ens which stencils only differ in the horizontal discretization, we can assess theoretically how $\left|\mathcal{T}_{\zeta}^{\text {een }}\right| /\left|\mathcal{T}_{\zeta}^{\text {ens }}\right|$ vary with $R_{U}$ and $R_{\Delta U}$. For this purpose, given $R_{U}$ and $R_{\Delta U}$, we estimate $\left|\mathcal{T}_{\zeta}^{\text {een }}\right| /\left|\mathcal{T}_{\zeta}^{\text {ens }}\right|$ by ensemble averaging over 10000 realizations of a random velocity field with prescribed parameters $R_{U}$ and $R_{\Delta U}$. The terms $\mathcal{T}_{\zeta}$ are computed with the same stencils as in the model. For given $R_{U}$ and $R_{\Delta U}$, the random two-dimensional velocity field $\mathcal{V}$ is defined on the grid illustrated in Fig. 10 by

$$
\mathcal{V}(\mathbf{r})=\widetilde{D}_{0}+R_{U}\left(\widetilde{D}_{1} \cdot \mathbf{r}+R_{\Delta U} \widetilde{N}(\mathbf{r})\right)
$$

where $\widetilde{D}_{0}$ is a $2 \times 1$ random vector, $\widetilde{D}_{1}$ is a $2 \times 2$ random tensor and $\widetilde{N}$ is a $2 \times 1$ random function of $\mathbf{r}$. All the random variables are normal variables with unit mean and .25 standard deviation. We found that the magnitude of the ratio computed below depends on the standard deviation of the random variables but its variations with $R_{U}$ and $R_{\Delta U}$ are robust.

[Fig. 10 about here.]

Fig. 11 shows the estimate of $\left|\mathcal{T}_{\zeta}^{\text {een }}\right| /\left|\mathcal{T}_{\zeta}^{\text {ens }}\right|$ as a function of $R_{U}$ and $R_{\Delta U}$ obtained with this random variable approach.

[Fig. 11 about here.]

First, it is striking that $\left|\mathcal{T}_{\zeta}^{\text {een }}\right|$ is systematically smaller than $\left|\mathcal{T}_{\zeta}^{\text {ens }}\right|$. Second, as expected, the mismatch between $\left|\mathcal{T}_{\zeta}^{\text {een }}\right|$ and $\left|\mathcal{T}_{\zeta}^{\text {ens }}\right|$ decreases with decreasing 
$R_{\Delta U}$. Third, the mismatch increases with increasing $U$. Indeed, $\left|\mathcal{T}_{\zeta}^{\text {een }}\right| /\left|\mathcal{T}_{\zeta}^{\text {ens }}\right| \rightarrow$ 1 with increasing $R_{U}$, either for a fixed $R_{\Delta U}$ or for a fixed $\delta^{2} U / U$ (see the hyperbola on Fig. 11).

\subsection{Diagnosing the regularity of the velocity field at the grid scale}

Having described the variations of $\left|\mathcal{T}_{\zeta}^{\text {een }}\right| /\left|\mathcal{T}_{\zeta}^{\text {ens }}\right|$ with $R_{U}$ and $R_{\Delta U}$, we can estimate these parameters in the model runs. This will help to find the dominant effect responsible for the mismatch between the momentum advection terms. The right panel of Fig. 12 presents an estimate of $R_{\Delta U}$ in run EEN, ENS and EFX. This estimate is obtained by comparing the relative vorticity $\zeta$ with its nine-points spatial average $\bar{\zeta}^{l o c}$ in order to define a typical scale of the grid-scale velocity gradient variations $\delta \zeta=\left|\zeta-\bar{\zeta}^{l o c}\right|$. Then, $\zeta / \delta \zeta$ is averaged over levels of constant $k_{u p}$ which yields, for each $k_{u p}$, the following estimate

$$
R_{\Delta U}=<|\delta \zeta| /|\zeta|>_{k_{u p}=c s t}
$$

Although we do not provide any formal proof that such an estimate allows to recover the prescribed parameters for a velocity field obtained from eq. (13), this estimate compares the amplitude of the grid scale variation of the velocity gradients with the velocity gradients themself. This estimate is drawn as a function of $k_{u p}$ in the the right panel of Fig. 12. Noticeably, $R_{\Delta U}$ appears to increase significantly close to the bottom topography in the three runs EEN, ENS and EFX, ranging from .25 in the domain interior to .65 at the lowermost levels. Moreover, it appears that $R_{\Delta U}$ is slightly enhanced in run EFX and reduced in run EEN. 
For the left panel of Fig. 12, an analogous procedure has been followed in order to estimate $R_{U}$.

[Fig. 12 about here.]

The norm of the velocity field $\|\mathbf{u}\|$ is compared with its nine-points spatial average yielding a typical velocity scale and a typical scale of the grid-scale velocity variations. Their ratio is then averaged over levels of constant $k_{u p}$ which gives a measure of $\delta U / U$ for each $k_{u p}$. This ratio is presented in the left panel of Fig. 12. Here again, it appears that $R_{U}$ increases close to the bottom topography. Considering Fig.11, such an increase of $R_{U}$ should tend to increase the ratio $\left|\mathcal{T}_{\zeta}^{e e n}\right| /\left|\mathcal{T}_{\zeta}^{\text {ens }}\right|$ in bottom layers which is not consistent with Fig. 8. We therefore conclude that the dominant effect responsible for the mismatch between $\left|\mathcal{T}_{\zeta}^{\text {een }}\right|$ and $\left|\mathcal{T}_{\zeta}^{\text {ens }}\right|$ in bottom layers is the observed increase of $R_{\Delta U}$ close to the bottom topography. In addition, the variation of $R_{\Delta U}$ from run to run is consistent with the increase of the mismatch with the ef $x$ scheme and decrease with the een scheme diagnosed in Fig. 8.

A complementary information is provided by Fig. 13 which presents an estimate of the irregularity of the vorticity tendency as computed by the three schemes along the same model run (EFX). Once again, this quantity is obtained by comparing $\mathcal{T}_{\zeta}$ with its nine-points spatial average and averaging over levels of constant $k_{u p}$.

[Fig. 13 about here.]

Fig. 13 shows that, $(i)$ the vorticity tendency gets more irregular in the bottom layers (see the left panel), (ii) with the same input fields, the een scheme (efx scheme) tends to produce a more (less) regular vorticity tendency than the 
ens scheme (see the right panel).

\subsection{Sensitivity to momentum advection schemes : conjectured mechanism}

At this point, we can conjecture the dynamical origin of the observed mean flow differences from run to run. Indeed, our study reveals that $(i)$ the momentum advection terms differ close to the topography in bottom layers due to an intensification of the grid-scale noise; $(i i)$ the level of grid scale noise is modified by the momentum advection scheme; (iii) a low level of grid scale noise close to the topography in bottom layers is concomitant with more intense deep currents; (iv) more intense deep currents coincide with improved mean surface circulation patterns. We therefore conjecture that the divergence of the model runs originates close to the topography and is related to the ability of the scheme to deal with under-resolved flows. We furthermore suggest that the schemes tend in the first place to modify the deep currents which in turn affect the upper layers flows. Althougth we cannot rule out a purely horizontal mechanism for the change in the upper layers flows, this second conjecture is fully supported by the study of Penduff et al. (2007). Penduff et al. (2007) have indeed found with a global $1 / 4^{\circ}$ ocean model that the changes in mean and eddy circulations from scheme ens to scheme een was vertically coherent and bottom intensified. This result, which has been verified with our North Atlantic model NATL025 (not shown), strongly supports that the change in the bottom and surface flows are dynamically connected and possibly due to a coupling of the bottom and surface flows. The picture which emerges from our study of the mechanism involved in the sensitivity to momentum advection schemes is schematically presented in Fig. 14. 
[Fig. 14 about here.]

Still, this diagram raises some fundamental issues.

First, what is the origin of the bottom intensification of the grid scale noise? Obviously, the proximity of the bathymetry plays an important role. Indeed, the treatment of the bottom boundary conditions are likely to produce gridscale perturbations of the vertical velocity field. This in turn can affect the temperature and salinity fields and eventually yield grid-scale perturbations in the horizontal pressure gradient. This would ultimately affect the horizontal velocity field. The side-wall boundary conditions in the bottom cells are also likely to induce grid-scale perturbations in the horizontal velocity field. We furthermore note that the treatment of the side-wall boundary conditions is not rigorously equivalent depending on the scheme (L. Debreu, personal communication, 2006). This may contribute to the different levels of gridscale noise observed in Fig. 12. Another possible effect is related to the weak stratification in the bottom layers which can decrease the horizontal scales of motions in the deep ocean (consider e.g. the reduced gravity Rossby radius associated with the Deep Western Boundary Current) and thus enhance the under-resolved character of the deep flow.

Second, how can the momentum advection terms and the grid scale noise affect the mean deep currents ? Two distinct effects can come into play in this respect. On the one hand, the different levels of grid-scale noise observed in the three simulations are likely to yield different amounts of explicit dissipation, that is, the explicit momentum diffusion operators extract more energy from the mean flow when the velocity field is more irregular. On the other hand, the mismatch in the bottom layers between the momentum advection 
terms computed with the three schemes (see Fig. 8 and Fig. 9) suggests that the implicit truncations errors should vary depending on the chosen scheme. We stress that, when discretizing a nonlinear term, the order of the residual truncation error can be high (see Minion and Brown, 1997). This may induce energy exchanges between the resolved scales of motions (Drikakis and Smolarkiewicz, 2001) (see the discussion in section 6).

Third, what is the dynamical link between the deep currents and the mean surface circulation ? We suggest that more intense deep currents allow for more vigorous bottom intensified eddy motions (including topographic Rossby waves). This hypothesis is corroborated by the work Penduff et al. (2007) (see their Fig.8) These more vigorous eddy motions in turn can interact with the surface intensified eddy field, resulting in a modification of the mean flow forcing by the surface eddy field. However, we emphasize that complete answers to the above questions goes beyond the scope of the present paper and would require further study. We nonetheless stress that the overall picture drawn in Fig.14 is corroborated by the work of Penduff et al. (2007) who address similar questions from a complementary perspective.

\section{Conclusion and discussion}

\subsection{Summary}

In this paper, we have studied the impact of the Coriolis-momentum advection scheme on eddy-admitting ocean simulations in a realistic context. Three sensitivity simulations have been performed in the North-Atlantic $1 / 4^{\circ}$ DRAKKAR model configuration. Three second order Coriolis-momentum ad- 
vection schemes conserving respectively enstrophy (ens), energy (efx) and both quantities (een) have been tested. The vertical dependence of the MKE profiles, usually overestimated in eddy-admitting ocean models (Penduff et al., 2005, 2007), has been shown to be reduced with the een scheme and enhanced with the efx scheme relative to the ens scheme. Concurrently, usual biases in the circulation patterns in the western North-Atlantic are significantly reduced with the een scheme.

In order to investigate the origin of this sensitivity, the behavior of the Coriolismomentum advection schemes in the three runs has been analyzed. We have shown that the Coriolis-momentum advection schemes differ mostly in the lowermost levels of the computational domain where their averaged contribution to the vorticity tendency is reduced with the een scheme and enhanced with the $e f x$ scheme. The comparison of these terms as computed along the same model solution and along separate runs reveals that the mismatch between the Coriolis-momentum advection terms as computed by the three schemes is due to the instantaneous tendency of each scheme.

Furthermore, it has been shown that the change in the regularity of the velocity field (i.e. the smoothness of the velocity gradients) close to the topography in bottom layers is responsible for the mismatch between the een scheme and the ens scheme (and most probably for the scheme ef $x$ as well). The mismatch between the een scheme and the ens scheme has been shown to depend on two parameters : $(i)$ the relative magnitude of the grid-scale velocity gradients $R_{U}$, and (ii) the regularity of the velocity gradients at the grid scale $R_{\Delta U}$. The latter effect dominates the variation of the Coriolis-momentum advection term in our simulations. In addition, the een scheme is shown to reduce the grid scale noise close to the topography in bottom layers whereas the ef $x$ scheme 
tends to increase the grid-scale noise.

These results allows one to conjecture that the differences of the mean flow fields in the three runs are related to the ability of the Coriolis-momentum advection scheme to deal with under-resolved flows close to the topography in bottom layers and sustain more intense mean and eddy currents at depth which in turn modify the surface flow. This work emphasizes the critical influence of current-topography interactions on the realism of basin scale circulations in ocean climate models.

\subsection{Discussion}

From a practical standpoint, the present study suggests that using the een

scheme in a C-grid, z-coordinate ocean model at eddy-permitting resolution can substantially improve the realism of the simulations. However, this statement should be mitigated by considering the limitations of the material presented herein. Indeed, the focus of this paper was put on the mechanism responsible for the sensitivity of the model and not on the quality of the solutions. Furthermore, the mechanism investigated in this study is probably not the only effect of the een scheme on the model solutions. In particular, we have not discussed the treatment of the lateral boundary conditions which, depending on the scheme is known to affect the basin scale vorticity budget in idealized configurations (Dupont et al., 2003). Likewise, we focused our diagnostics on the representation of current-topographic interactions. The mechanism described above is therefore not relevant for regions where the flow regime is not directly dependant on current-topographic interactions. As an example, we have not discussed the effect of Coriolis-momentum advection schemes in 
equatorial regions. Another limitation of this study is that our comparisons are restricted to second order numerical schemes. We nonetheless stress that even if higher order schemes are known to be less sensitive to grid-scale noise, their spatial order is usually degraded close to the boundaries in order to handle the boundary conditions. The sensitivity to Coriolis-momentum advection schemes should therefore be significant in the bottom layer for higher order schemes as well. Besides those limitations, we recommend the use of the een scheme among the second order schemes available in NEMO for basin-scale simulations at eddy-admitting resolutions, especially for mid-latitude eddyactive regions. In addition, we stress that the high sensitivity to momentum advection schemes in bottom layers will probably appear at higher horizontal resolution as well. Indeed, increasing the horizontal resolution yields an increase of the mean deep currents. But section 5.1 has shown that the mismatch between the schemes increases with increasing velocity. We therefore believe that the sensitivity in bottom layers can also be significant at higher resolutions even though the flows are better resolved. But a clean comparison of the schemes at higher resolution is needed to confirm this point.

Important questions remain on the actual link between the Coriolis-momentum advection scheme and the intensification of deep-currents. We acknowledge that further work is required to distinguish between the role of the explicit dissipation and the implicit truncation errors in this respect. More precisely, it would be valuable to examine the energy actually dissipated by the dissipation operators in the simulations and to check analytically the structure of the truncation errors for the three schemes. This latter task may be quite cumbersome due to the complexity of the spatial stencils involved. We nonetheless stress that the truncation of nonlinear terms may yield coherent modifications 
of the resolved flow at large scale and not only an equivalent dissipation or dispersion (see e.g. Minion and Brown, 1997). These effects (including the so called "spurious vortices" in the incompressible two-dimensional dynamics) are related to an erroneous nonlinear coupling between resolved scales of motions via the nonlinear truncation error (Drikakis and Smolarkiewicz, 2001). Depending on the scheme, spurious interior vorticity sources may arise and yield a direct modification of the resolved scales of motions. In this context, the fact that Arakawa and Lamb (1981) observed that the een scheme can conserve more accurately potential vorticity might be a indirect indication that the een scheme is less likely to produce spurious interior vorticity sources.

An important conclusion of this work is the critical influence of current topography interactions on basin scale surface circulation. Keeping this in mind might provide a guideline for further improvements of ocean-climate models. Indeed, in their paper, Penduff et al. (2007) noted that the use of the een scheme yields a $20 \%$ increase of the mean currents in the deep ocean in the global $1 / 4^{\circ}$ DRAKKAR model. Another $20 \%$ increase was also obtained if a partial step topography was used in addition. Besides, it is striking that partial steps have been shown to reduce significantly the grid-scale noise close to the bottom topography (Pacanowski and Gnanadesikan, 1998). The results of the present study suggest that the sensitivity of the mean currents to momentum advection schemes and partial steps takes similar routes, that is, a reduction of the grid-scale noise in bottom layers and, supposedly, a subsequent reduction of the explicit dissipation in the deep ocean. This remark raises the issue of the crucial sensitivity of eddying ocean models regarding the representation of the bottom topography. In particular, it might be useful to remove grid scale features from ocean model topographies over which current-topography 
interactions are not resolved.

\section{Acknowledgments}

The DRAKKAR project team is gratefully acknowledged. We thank J.-M. Molines for his valuable advice, A.-M. Treguier for her constructive comments and suggestions and L. Debreu for his helpful remarks. We also would like to thank two anonymous reviewers, whose comments have helped and improved a previous version of this article. Technical support from the NEMO team was also appreciated. The simulations were performed at IDRIS/CNRS on a NEC SX-5 supercomputer. This work is a contribution to the MERSEA project. Partial support from the European Commission under Contract SIP3-CT2003-502885 is gratefully acknowledged.

\section{References}

Adcroft A., C. Hill, J.-M. Campin, J. Marshall and P. Heimbach. Overview of the Formulation and Numerics of the MIT GCM. Proceedings of the ECMWF seminar series on Numerical Methods, Recent developments in numerical methods for atmosphere and ocean modelling. 139-149, 2004.

Adcroft A., C. Hill and J. Marshall. Representation of Topography by Shaved Cells in a Height Coordinate Ocean Model. Monthly Weather Review 125:2293-2315, 1997.

Arakawa A. and V. Lamb. A potential enstrophy and energy conserving scheme for the shallow water equations. Monthly Weather Review, 109:18-136, 1981.

Barnier B., G. Madec, T. Penduff, J.-M. Molines, A.-M. Treguier, J. Le Sommer, A. Beckmann, A. Biastoch, C. Bning, J. Dengg, S. Gulev, C. Derval, E. Du- 
rand, E. Remy, C. Talandier, S. Theetten, M. Maltrud, J. McClean and B. De Cuevas. Impact of partial steps and momentum advection schemes in a global ocean circulation model at eddy permitting resolution. Ocean Dynamics,in press, 2006.

Beckmann A. and R. Döscher. A method for improved representation of dense water spreading over topography in geopotential-coordinate models. Journal of Physical Oceanography, 27:581-591, 1997.

Bleck R., C. Rooth, D. Hu and L.T. Smith. Ventilation patterns and mode water formation in a wind- and thermodynamically driven isopycnic coordinate model of the North Atlantic. Journal of Physical Oceanography 22:1486-1505,1992.

Bryan, K.. A numerical method for the study of the circulation of the world ocean. Journal of Computational Physics, 4:347-376, 1969.

Bryan, K.. A scheme for numerical integration of the equations of motions on an irregular grid free of nonlinear instability. Monthly Weather Review 94:39-40, 1966.

Burridge D.M. and J. Haseler. A model for medium range weather forecasting - Adiabatic formulation. Tech. Rep., 4, European Center for Medium Range Weather Forecasts, Bracknell, Berks., UK, 46pp, 1977.

Candela J., S. Tanahara, M. Crepon, B. Barnier and J. Sheinbaum. Yucatan Channel flow: observations versus Clipper Atl6 and Mercator Pam models. Journal of Geophysical Research 108 (C12), 3385, 2003

Chassignet, E.P., H.G. Arango, D. Dietrich, T. Ezer, M. Ghil, D.B. Haidvogel, C.-C. Ma, A. Mehra, A.M. Paiva and Z. Sirkes. DAMEE-NAB: The Base Experiments. Dynamics of Atmospheres and Oceans, 32:155-183, 2000.

Drikakis D. and P.K. Smolarkiewicz On spurious vortical structures. Journal of Computational Physics, 172:309-325, 2001.

Dupont F., D.N. Straub and C.A. Lin. Influence of a step-like coastline on the basin scale vorticity budget of mid-latitude gyre models. Tellus, 55A:255-272, 2003. 
Fichefet T. and M.A. Morales Maqueda. Sensitivity of a global sea ice model to the treatment of ice thermodynamics and dynamics. Journal of Geophysical Research, 102:12609-12646, 1997.

Gent, P.R. and J.C. McWilliams. Isopycnal mixing in ocean circulation models. Journal of Physical Oceanography, 20(1):150-155, 1990

Gent, P.R., J. Willebrand, T.J. McDougall and J.C. McWilliams. Parameterizing Eddy-Induced Tracer Transports in Ocean Circulation Models. Journal of Physical Oceanography, 25(4):463-474, 1995.

Gerdes R., C. Kberle and J. Willebrand. The influence of numerical advection schemes on the results of ocean general circulation models. Climate Dynamics, 5(4):211-226, 1991.

Griffies S.M., A. Gnanadesikan, R.C. Pacanowski, V.D. Larichev, J.K. Dukowicz and R.D. Smith. Isoneutral Diffusion in a z-Coordinate Ocean Model. Journal of Physical Oceanography, 28(5):805:830, 1998.

Griffies S.M., C. Böning, F.O. Bryan, E.P. Chassignet, R. Gerdes, H. Hasumi, A. Hirst, A.-M. Treguier and D. Webb. Developments in ocean climate modelling. Ocean Modelling, 2:123:192, 2000.

Hallberg R.W. Stable split time stepping schemes for large-scale ocean modelling. Journal of Computational Physics 135:54-65,1997.

Ishizaki H. and T. Motoi. Reevaluation of the Takano-Oonishi scheme for momentum advection on bottom relief in ocean models. Journal of Atmospheric and Oceanic Technology, 16:1994-2010, 1999.

Jamart B.M. and J. Ozer. Numerical boundary layers and spurious residual flows. Journal of Geophysical Research, 91(C9):10621-10632, 1986.

Jourdan D, Balopoulos E, Garcia-Fernandez MJ, Maillard C. Objective Analysis of Temperature and Salinity Historical Data Set over the Mediterranean Basin, IEEE, 1998.

Kalnay E, Kanamitsu M, Kistler R, Collins W, Deaven D, Gandin L, Iredell M, Saha 
S, White G, Woollen J, Zhu Y, Chelliah M, Ebisuzaki W, Higgins W, Janowiak J, Mo KC, Ropelewski C, Leetmaa A, Reynolds R and Jenne R. The NCEP/NCAR 40-year reanalysis project. Bulletin of the American Meteorological Society 77: 437-471, 1996.

Large, W. G. and J.C. McWilliams and S.C. Doney. Oceanic vertical mixing: a review and a model with a nonlocal boundary layer parameterization. Reviews of Geophysics, 32(4), 363-404, 1994.

LeVeque R.J. Numerical methods for conservation laws, Second edition. Birkhuser Verlag, 1992.

Levitus S, T.P. Boyer, M.E. Conkright, T. O'Brian, J. Antonov, C. Stephens, L. Stathopolos, D. Johnson and R. Gelfeld. World Ocean Database 1998. NOAA Atlas NESDID18, 1998.

Madec G., P. Delecluse, M. Imbard and C. Lévy. OPA 8.1 Ocean General Circulation Model reference manual. Note du Pôle de modélisation, Institut Pierre-Simon Laplace, N11, 91pp, 1998.

Madec G. NEMO reference manual, ocean dynamics component : NEMO-OPA. Note du Pôle de modélisation, Institut Pierre-Simon Laplace, N27, 2006.

Merryfield, W. J. and R. B. Scott. Bathymetric influence on mean currents in two high-resolution near-global ocean models. Ocean Modelling, in press, 2006.

Mesinger F. and A. Arakawa. Numerical methods used in atmospheric models. GARP Publication Series No. 17, ICSU/WMO, 1976.

Minion M.L. and D.L. Brown. Performance of under-resolved two-dimensional incompressible flow simulation, II. Journal of Computational Physics, 138:734-765, 1997.

Niiler P.P., N.A. Maximenko and J.C. McWilliams. Dynamically balanced absolute sea level of the global ocean derived from near-surface velocity observations. Geophysical Research Letters, 30(22):2164, 2003.

Nechaev D. and M. Yaremchuk. On the Approximation of the Coriolis Terms in 
C-Grid Models. Monthly Weather Review, 132:2283-2289, 2004.

Pacanowski, R. and A. Gnanadesikan. Transient response in a z-level ocean model that resolves topography with partial cells. Monthly Weather Review, 126:3248$3270,1998$.

Pacanowski, R.C. and S.M. Griffies. The MOM 3 Manual. Geophysical Fluid Dynamics Laboratory/NOAA, Princeton, USA, p 680, 1999.

Penduff T., B. Barnier, K. Branger and J. Verron. Comparison of near-surface mean and eddy flows from two numerical models of the South Atlantic Ocean. Journal of Geophysical Research, 106(C8), 16857-16867, 2001.

Penduff T., B. Barnier, J.-M. Molines, G. Madec. On the use of current meter data to assess the realism of ocean model simulations. Ocean Modelling, 7:11-45, 2005.

Penduff T., J. Le Sommer, B. Barnier, J.-M. Molines and G. Madec. Influence of numerical schemes on current topography interactions in $1 / 4^{\circ}$ global ocean simulations. Ocean Science, in revision, 2007.

Sadourny R. The dynamics of finite-difference models of the shallow water equations. Journal of the Atmospheric Sciences, 32:680-689, 1975.

Salmon R. Poisson-Bracket approach to the construction of energy-and-potentialenstrophy-conserving algorithms for the shallow water equations. Journal of the Atmospheric Sciences, 61:2016-2036, 2004.

Smith, R.D., M.E. Maltrud, F.O. Bryan and M.W. Hecht. Numerical simulation of the North Atlantic Ocean at 1/10 . Journal of Physical Oceanography 30:1532$1561,2000$.

Steele M, R. Morley and W. Ermold. PHC: A global ocean hydrography with a high quality Arctic Ocean. Journal of Climate 14: 2079-2087, 2001.

Webb D.J., B.A. de Cuevas and C.S. Richmond. Improved advection schemes for ocean models. Journal of Atmospheric and Oceanic Technology, 15:1171-1187, 1998.

Willebrand J., B. Barnier, C. Böning, C. Dieterich, P.D. Killworth, C. LeProvost, 
Y. Jia, J.-M. Molines and A.L. New. Circulation characteristics in three eddypermitting models of the North Atlantic. Progress in Oceanography, 48(2-3):123$161,2001$.

Xie P and P.A. Arkin. Global Precipitation: A 17-Year monthly analysis based on gauge observations, satellite estimates, and numerical model outputs. Bulletin of the American Meteorological Society 78(11): 2539-2558, 1997. 


\section{List of Figures}

1 Schematic of the stencils used to compute the term $(f+\zeta) v$ at $u$-points $(i, j+1 / 2)$ on a C-grid with the een scheme (left panel) and the ens scheme (right panel). Grey dots are located at $f$-points and black arrows at $u$-points. Thick lines indicate linear averaging of variables.

2 Computational domain and bottom topography (depth in $\mathrm{m}$ ) of the DRAKKAR-NATL025 configuration.

3 Mean sea surface height in the North Atlantic from Niiler et al. (2003) (upper left panel) and during year 8-10 of run EEN (upper right panel), run ENS (lower left panel), run EFX (lower right panel). In each plot, the mean area sea surface height has been subtracted.

4 Definition of $k_{u p}$ for $T$-points variables (left panel) and for $f, v$-points variables (right panel). Black dots indicate $T$-points.

5 Mean kinetic energy vertical profiles. The average has been performed on level of constant $k_{u p}$ (see Fig. 4). That is, each numerical value is obtained by averaging over points within a fixed distance from the bottom topography. Left panel : mean kinetic energy (unit $: \mathrm{m}^{2} \cdot \mathrm{s}^{-2}$ ) as a function of $k_{u p}$ (vertical axis) in run EEN (open circles), run ENS (black dots) and run EFX (open squares). Right panel : mean kinetic energy ratios with respects to run ENS. Recall that, as indicated in the text, the ens scheme has been chosen as a common reference for schemes een and efx.

6 Schematic of the diagnostic approach. Model solution are represented in the state vector space of the model. The arrows indicate the tendency due to the Coriolis-momentum advection term as computed by schemes ens, een and ef $x$.

$7 \quad$ Snapshot of the surface $\mathcal{T}_{\zeta}$ during the summer of year 11 . upper panel : surface $\mathcal{T}_{\zeta}$ in run EEN as computed with the scheme een. bottom panel : $\mathcal{T}_{\zeta}\left(\right.$ unit $: \mathrm{s}^{-2}$ ) on solution EEN along the black line of the upper panel, as computed with the scheme een (blue circles), the scheme ens (black circles) and the scheme efx (red circles). 
8 Left panel : $T\left(k_{u p}\right)$ as computed along each model solution (unit : $\left.10^{-12} \mathrm{~s}^{-2}\right)$. Right panel : ratios of $T\left(k_{u p}\right)$ as computed in model run EEN, ENS and EFX.

$9 \quad T\left(k_{u p}\right)$ as computed along the model solution EFX with schemes ens, een and ef $x$ (unit : $10^{-12} \mathrm{~s}^{-2}$ ).

10 Schematic of the grid used for the statistical estimation of $\left|\mathcal{T}_{\zeta}^{e e n}\right| /\left|\mathcal{T}_{\zeta}^{e n s}\right|$. The vector $\mathbf{r}$ denotes the coordinate on the grid. $\left|\mathcal{T}_{\zeta}^{\text {een }}\right|$ and $\left|\mathcal{T}_{\zeta}^{\text {ens }}\right|$ are computed at point $(0,0)$ for each realization of the random velocity field $[\mathcal{V}$, then averaged over 10000 realizations of $\left[\mathcal{V}_{\mathcal{V}}\right.$ in order to get the ratio $\left|\mathcal{T}_{\zeta}^{\text {een }}\right| /\left|\mathcal{T}_{\zeta}^{\text {ens }}\right|$

11 Theoretical estimate of the ratio $\left|\mathcal{T}_{\zeta}^{\text {een }}\right| /\left|\mathcal{T}_{\zeta}^{\text {ens }}\right|$ showing its variations with respects to $R_{U}$ and $R_{\Delta U}$. See the text for details.

12 Estimates of $R_{U}$ and $R_{\delta U}$ as a function of $k_{u p}$ during the summer of year 11 in run EEN, ENS and EFX (see the text for details).

13 Irregularity of $\mathcal{T}_{\zeta}$ the vorticity tendency due to horizontal advection as computed by schemes ens, een and ef $x$ during the summer of year 11 in run EFX.

14 Diagram synthesizing the conjectured mechanism responsible for the spreading of our three model runs. 


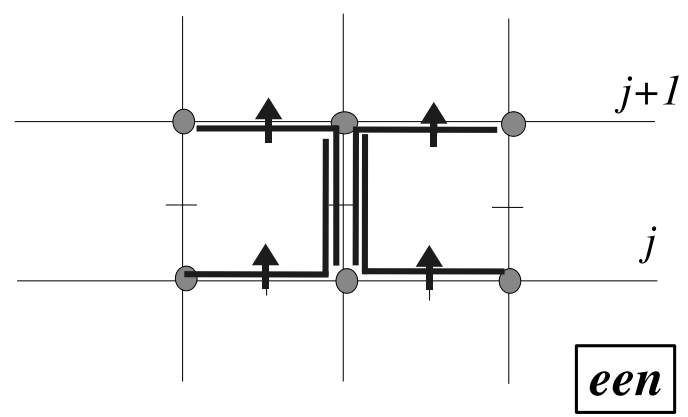

i-1

$i$

$i+1$

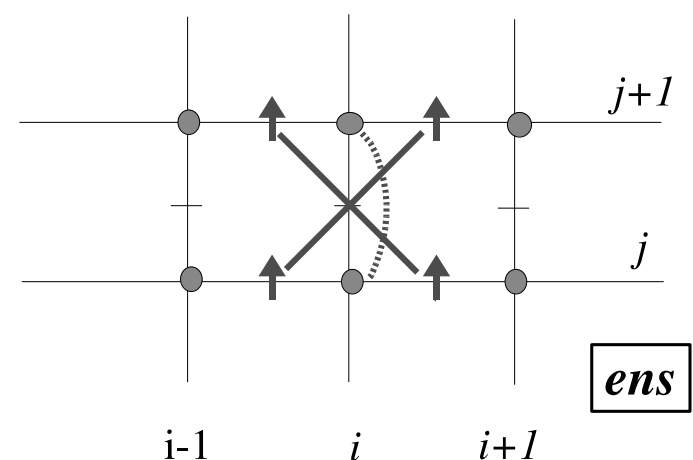

Fig. 1. Schematic of the stencils used to compute the term $(f+\zeta) v$ at $u$-points $(i, j+1 / 2)$ on a C-grid with the een scheme (left panel) and the ens scheme (right panel). Grey dots are located at $f$-points and black arrows at $u$-points. Thick lines indicate linear averaging of variables. 


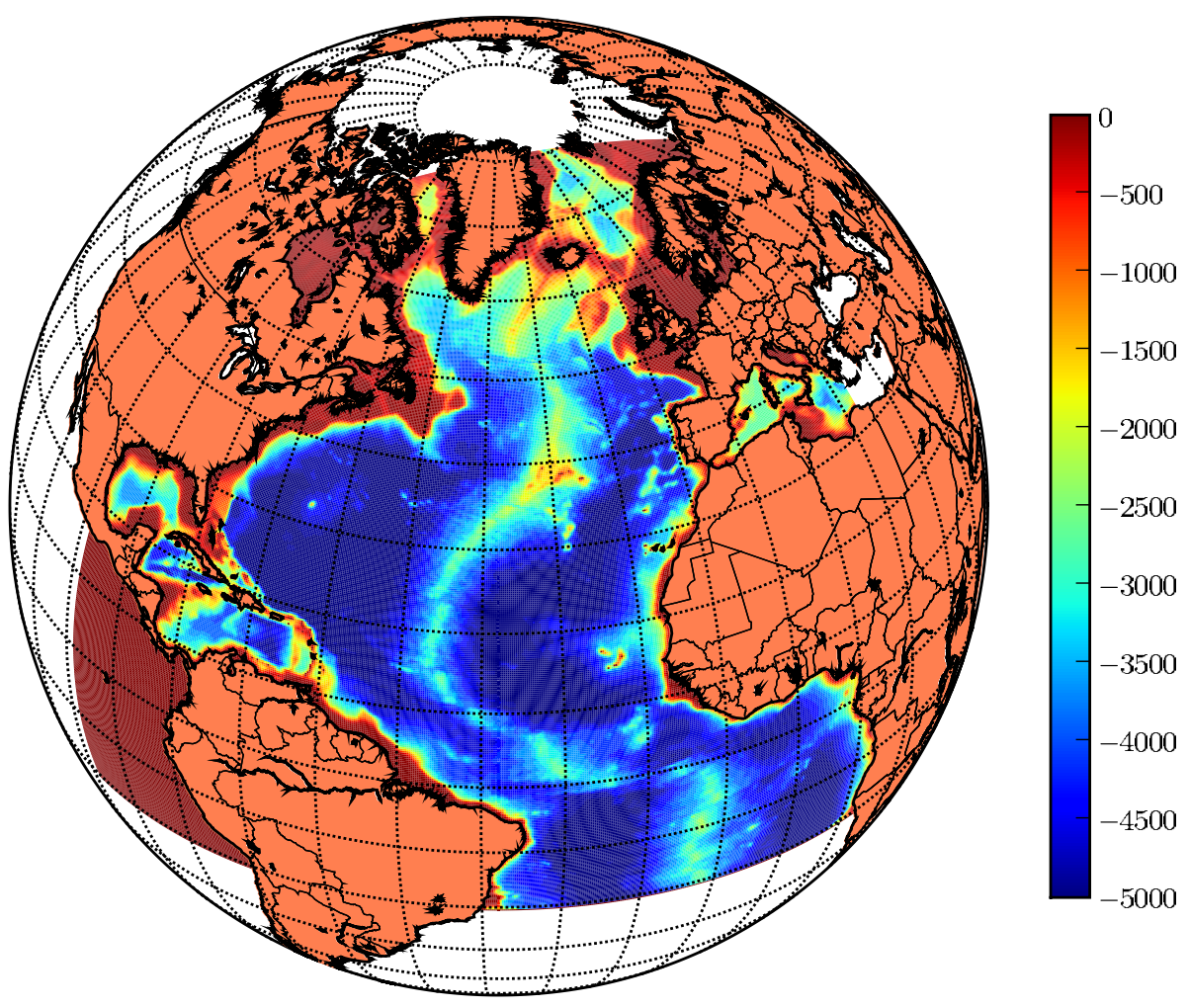

Fig. 2. Computational domain and bottom topography (depth in $\mathrm{m}$ ) of the DRAKKAR-NATL025 configuration. 

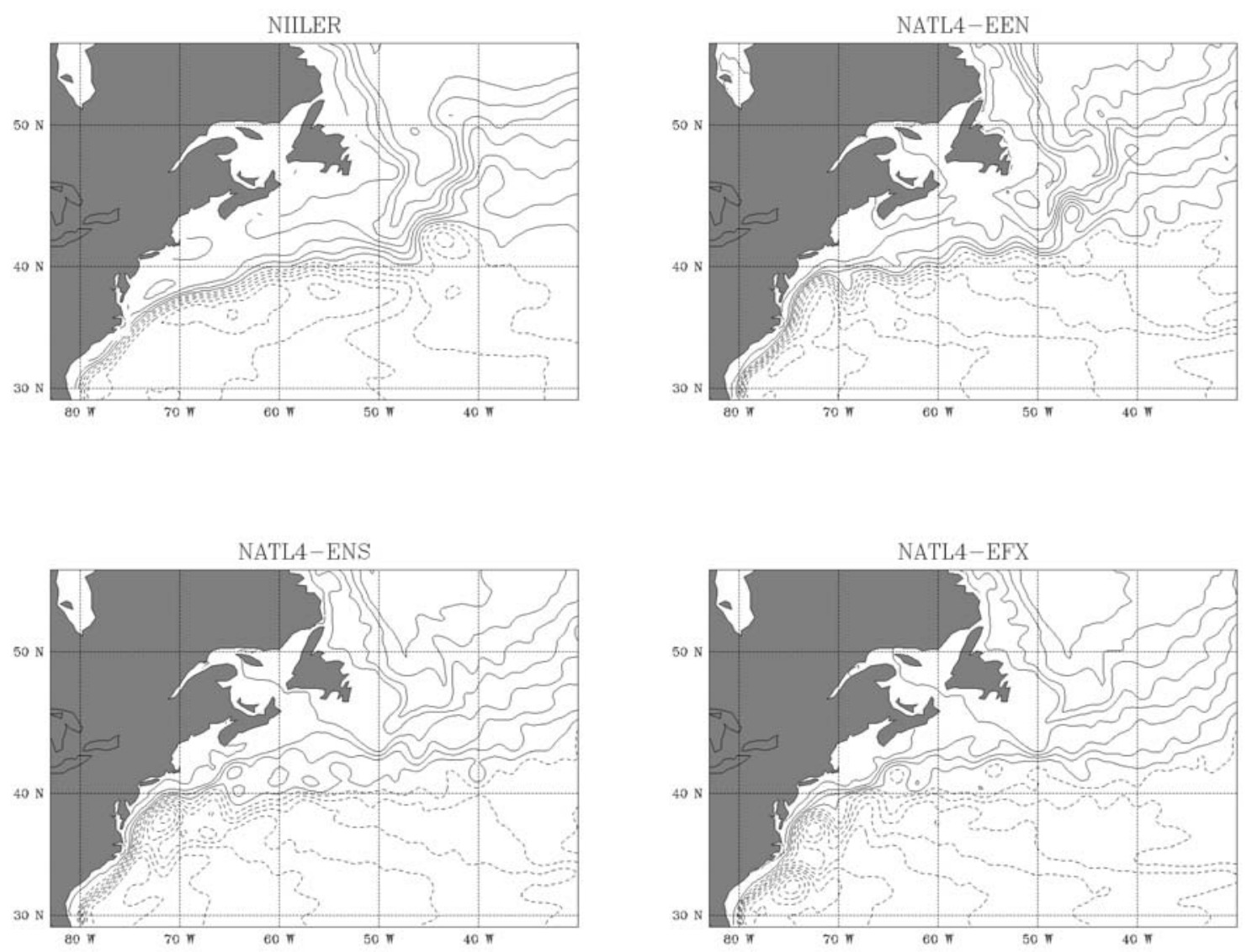

Fig. 3. Mean sea surface height in the North Atlantic from Niiler et al. (2003) (upper left panel) and during year 8-10 of run EEN (upper right panel), run ENS (lower left panel), run EFX (lower right panel). In each plot, the mean area sea surface height has been subtracted. 

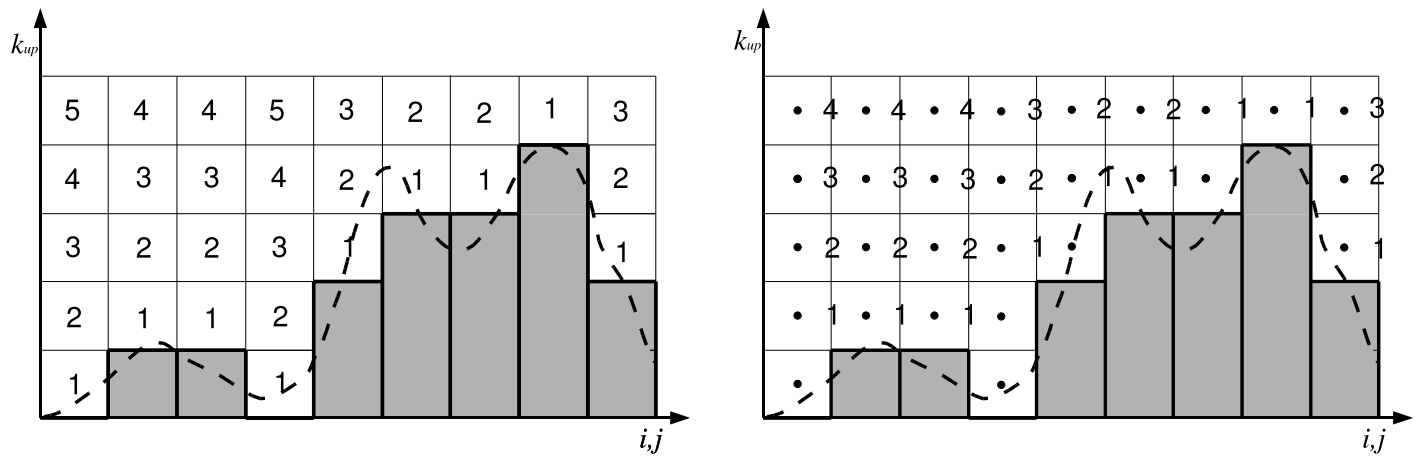

Fig. 4. Definition of $k_{u p}$ for $T$-points variables (left panel) and for $f, v$-points variables (right panel). Black dots indicate $T$-points. 

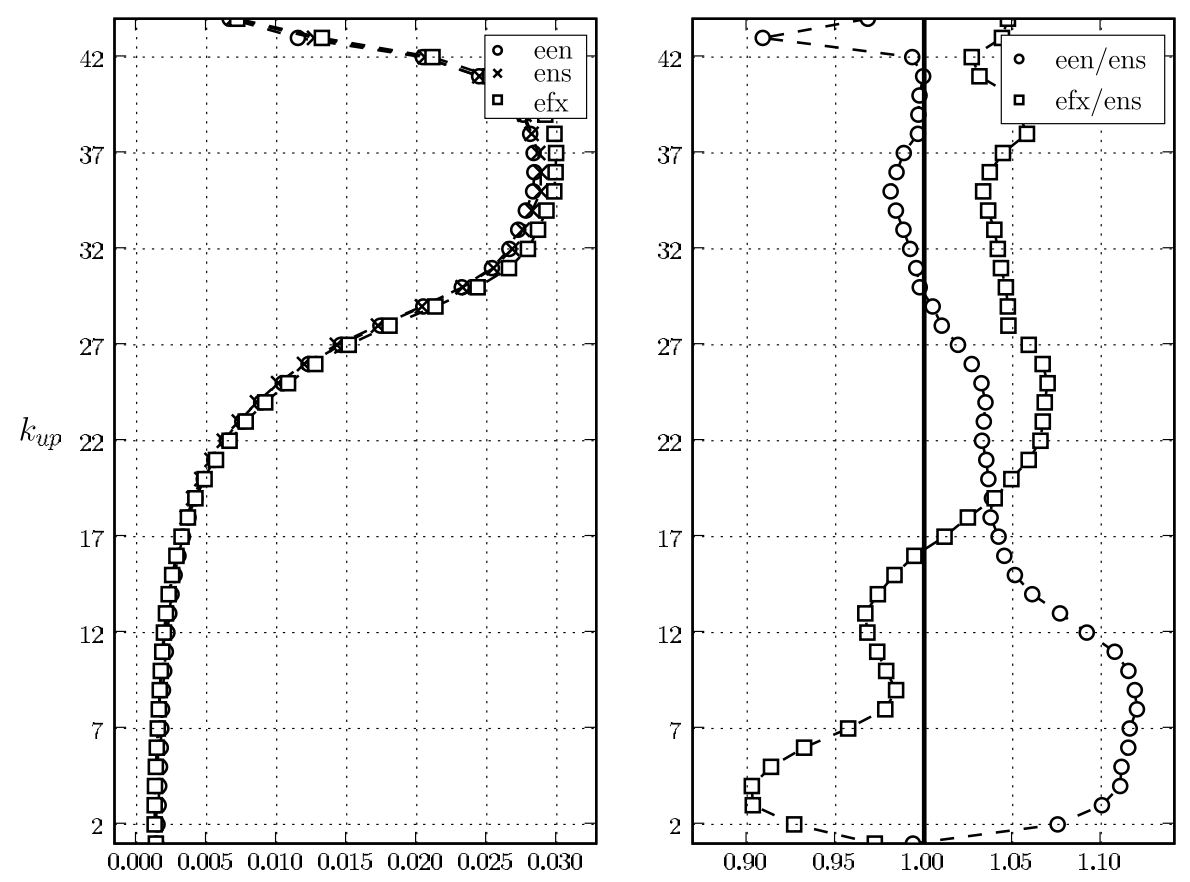

Fig. 5. Mean kinetic energy vertical profiles. The average has been performed on level of constant $k_{u p}$ (see Fig. 4). That is, each numerical value is obtained by averaging over points within a fixed distance from the bottom topography. Left panel : mean kinetic energy (unit $: \mathrm{m}^{2} . \mathrm{s}^{-2}$ ) as a function of $k_{u p}$ (vertical axis) in run EEN (open circles), run ENS (black dots) and run EFX (open squares). Right panel : mean kinetic energy ratios with respects to run ENS. Recall that, as indicated in the text, the ens scheme has been chosen as a common reference for schemes een and efx. 


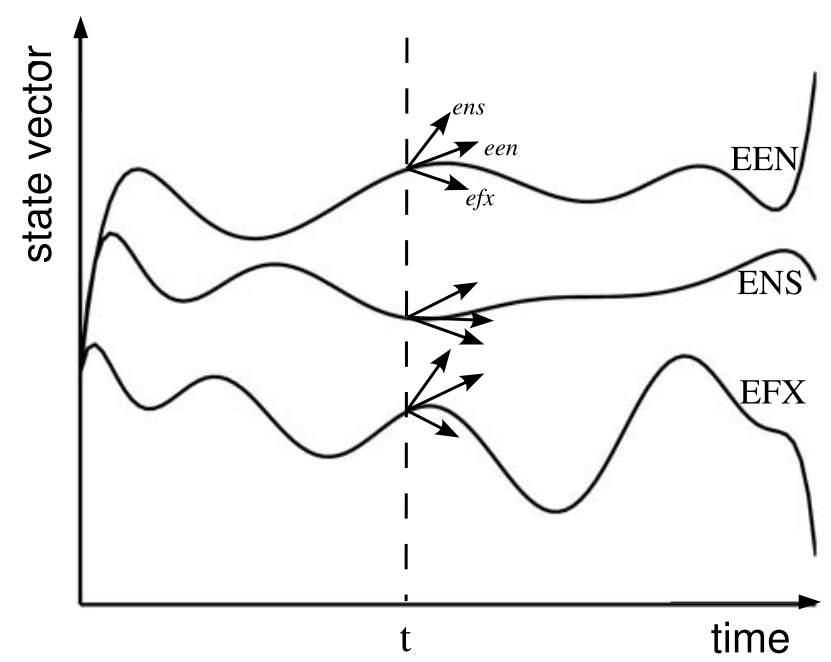

Fig. 6. Schematic of the diagnostic approach. Model solution are represented in the state vector space of the model. The arrows indicate the tendency due to the Coriolis-momentum advection term as computed by schemes ens, een and ef $x$. 

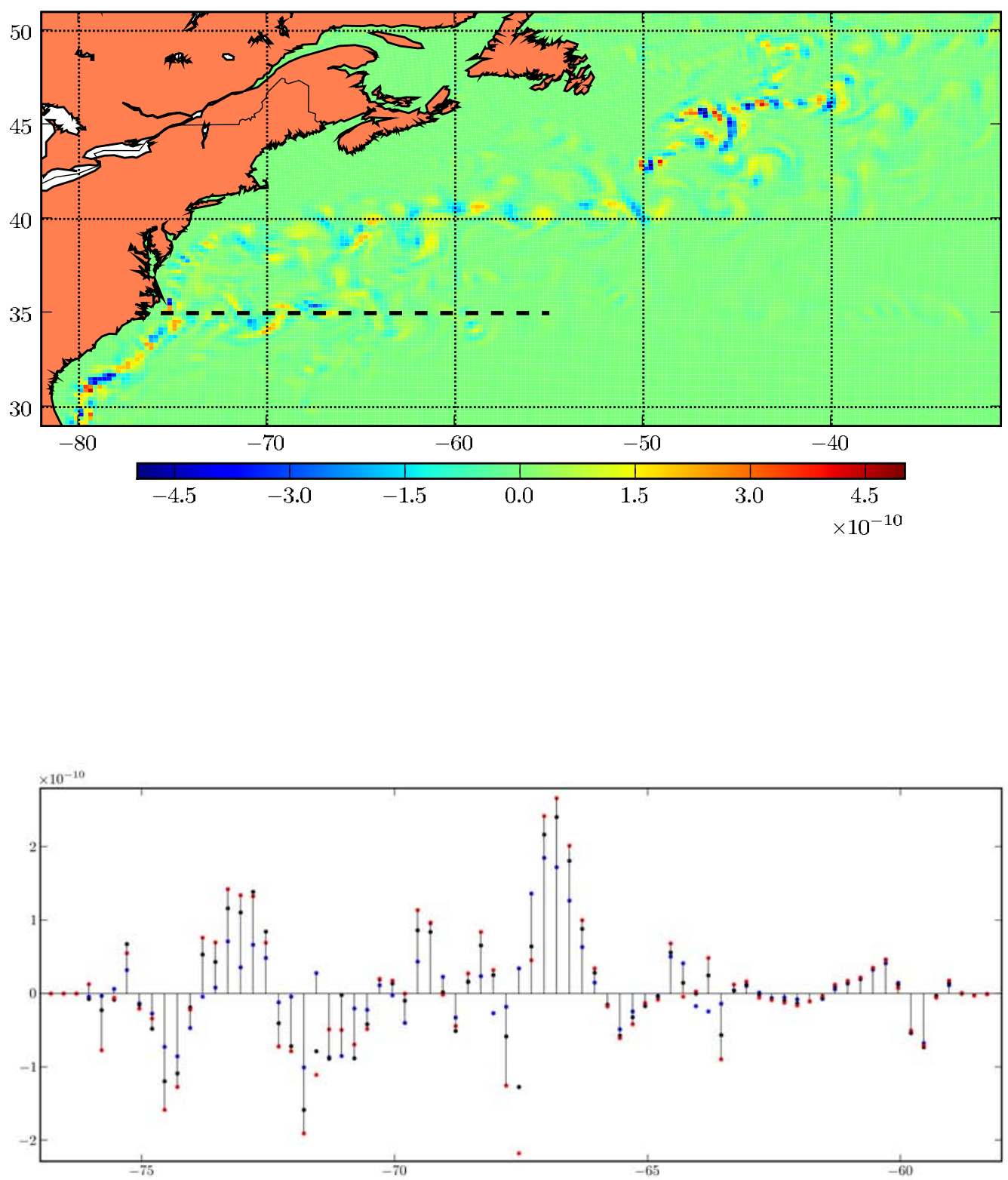

Fig. 7. Snapshot of the surface $\mathcal{T}_{\zeta}$ during the summer of year 11. upper panel : surface $\mathcal{T}_{\zeta}$ in run EEN as computed with the scheme een. bottom panel : $\mathcal{T}_{\zeta}$ (unit : $\mathrm{s}^{-2}$ ) on solution EEN along the black line of the upper panel, as computed with the scheme een (blue circles), the scheme ens (black circles) and the scheme efx (red circles). 

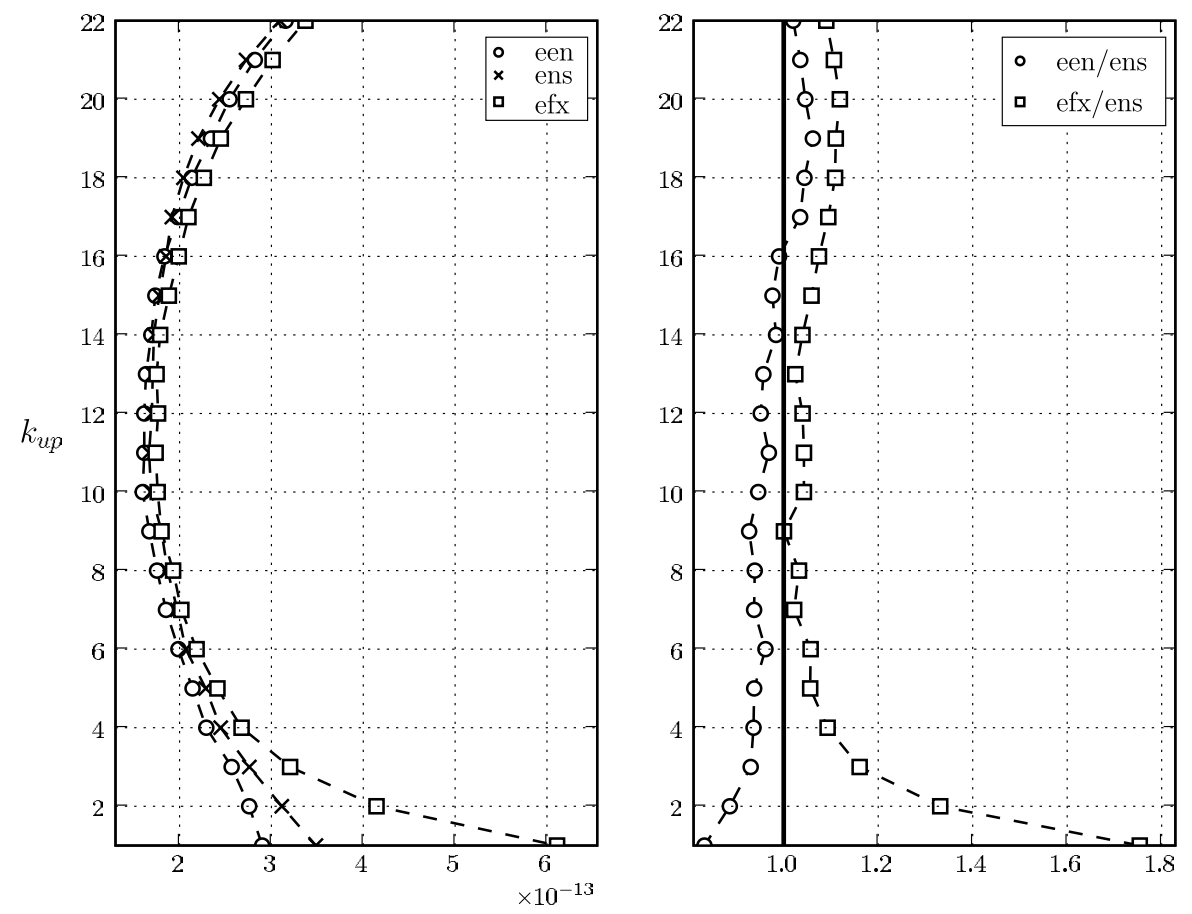

Fig. 8. Left panel : $T\left(k_{u p}\right)$ as computed along each model solution (unit : $10^{-12} \mathrm{~s}^{-2}$ ). Right panel : ratios of $T\left(k_{u p}\right)$ as computed in model run EEN, ENS and EFX. 

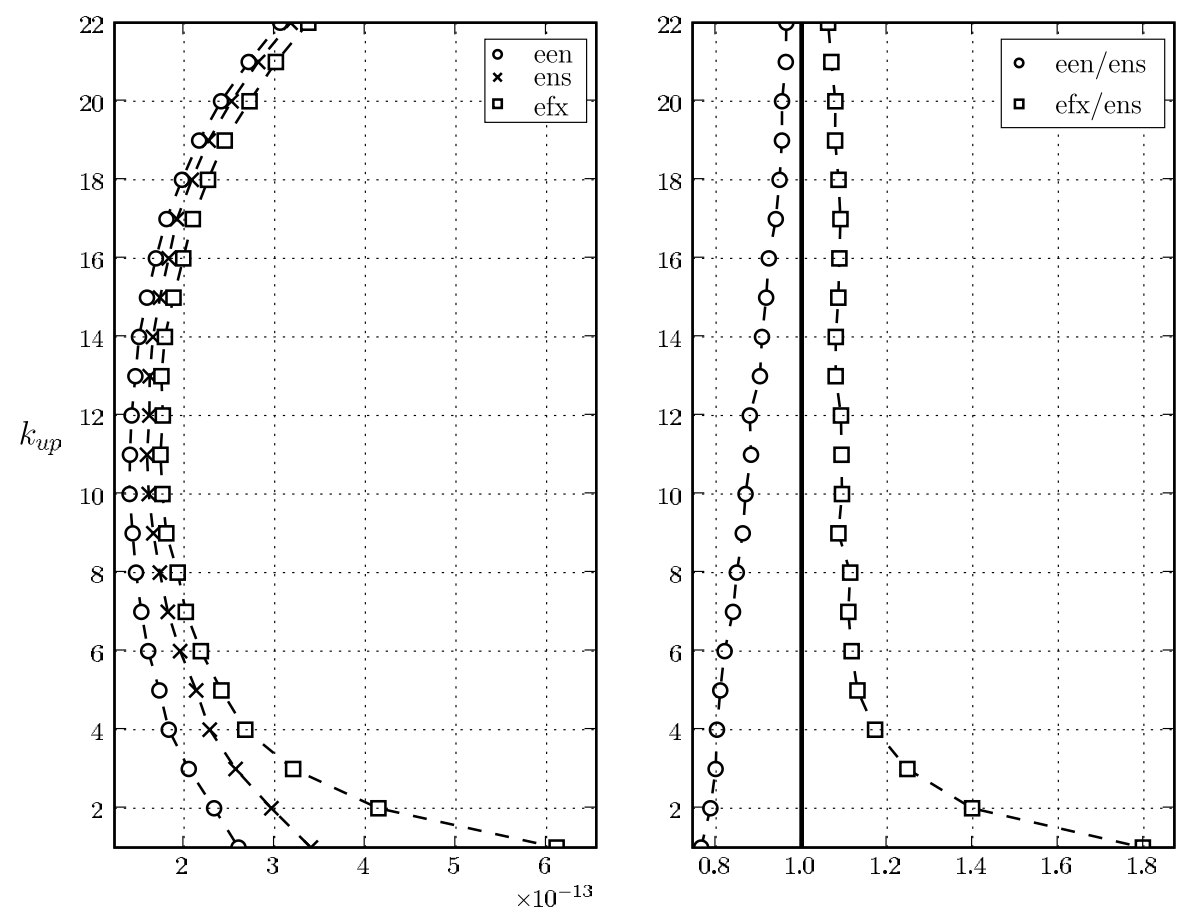

Fig. 9. $T\left(k_{u p}\right)$ as computed along the model solution EFX with schemes ens, een and ef $x$ (unit : $10^{-12} \mathrm{~s}^{-2}$ ). 


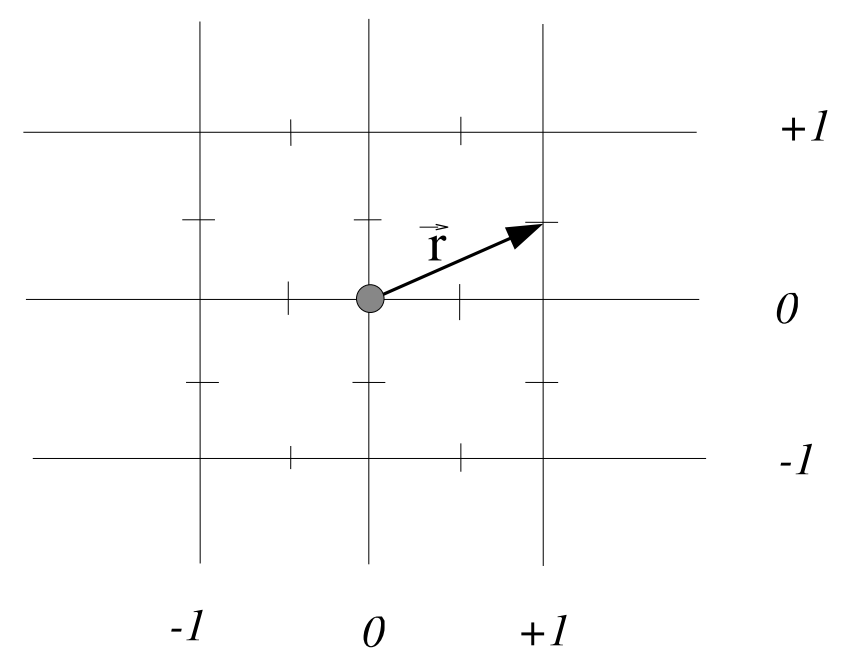

Fig. 10. Schematic of the grid used for the statistical estimation of $\left|\mathcal{T}_{\zeta}^{\text {een }}\right| /\left|\mathcal{T}_{\zeta}^{\text {ens }}\right|$. The vector $\mathbf{r}$ denotes the coordinate on the grid. $\left|\mathcal{T}_{\zeta}^{\text {een }}\right|$ and $\left|\mathcal{T}_{\zeta}^{\text {ens }}\right|$ are computed at point $(0,0)$ for each realization of the random velocity field $\underset{\sim}{\mathcal{V}}$, then averaged over

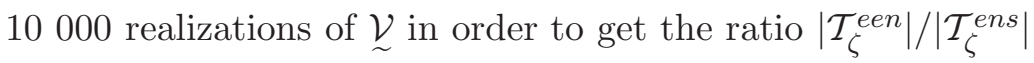




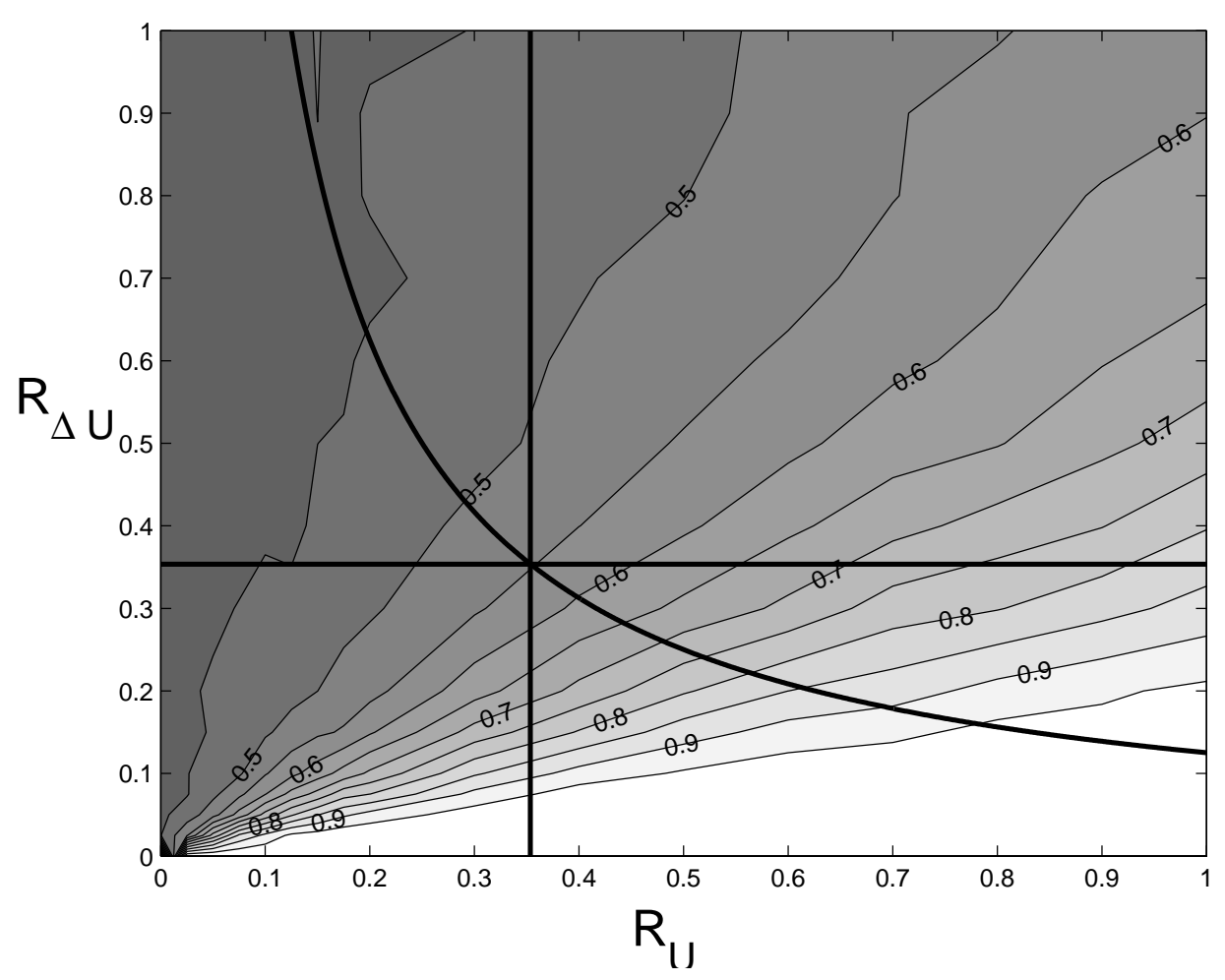

Fig. 11. Theoretical estimate of the ratio $\left|\mathcal{T}_{\zeta}^{\text {een }}\right| /\left|\mathcal{T}_{\zeta}^{\text {ens }}\right|$ showing its variations with respects to $R_{U}$ and $R_{\Delta U}$. See the text for details. 

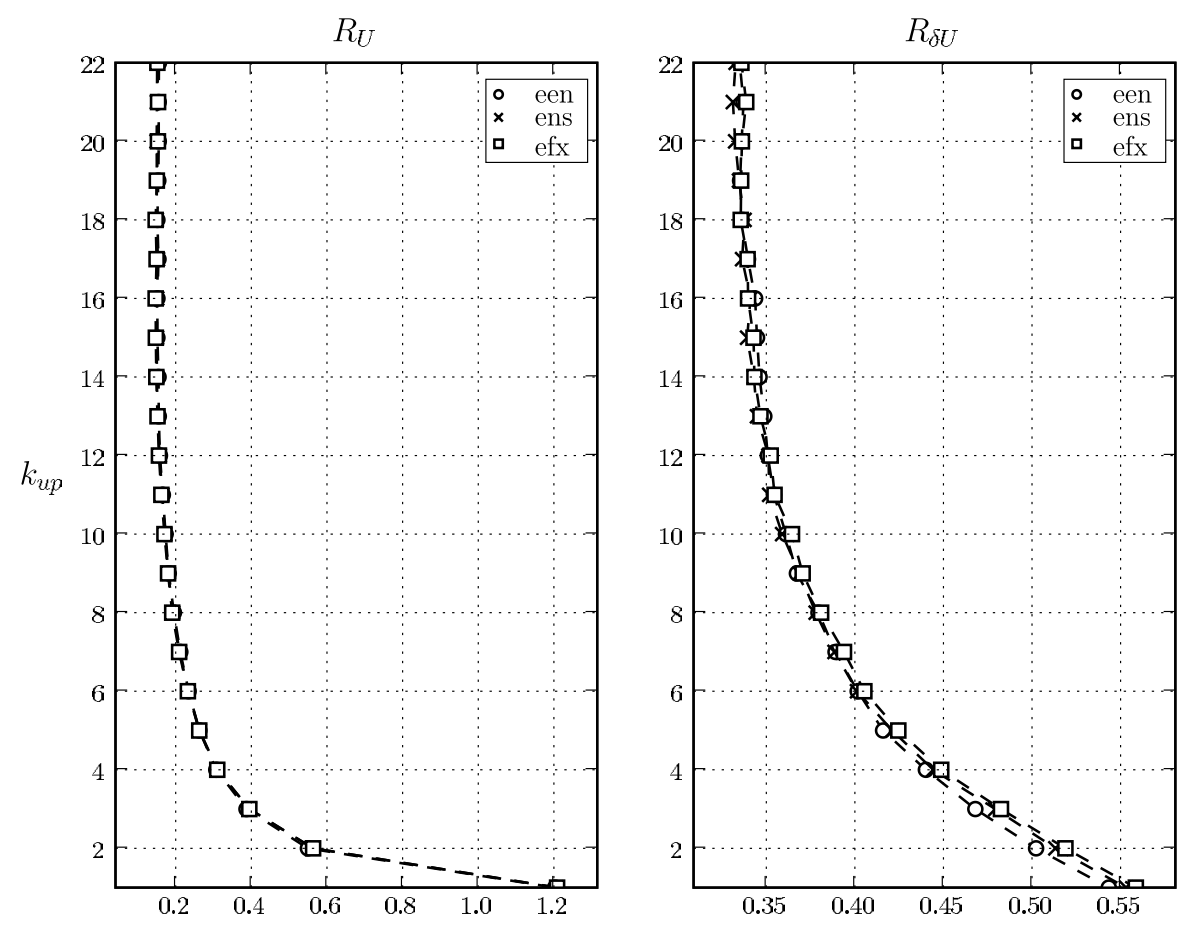

Fig. 12. Estimates of $R_{U}$ and $R_{\delta U}$ as a function of $k_{u p}$ during the summer of year 11 in run EEN, ENS and EFX (see the text for details). 

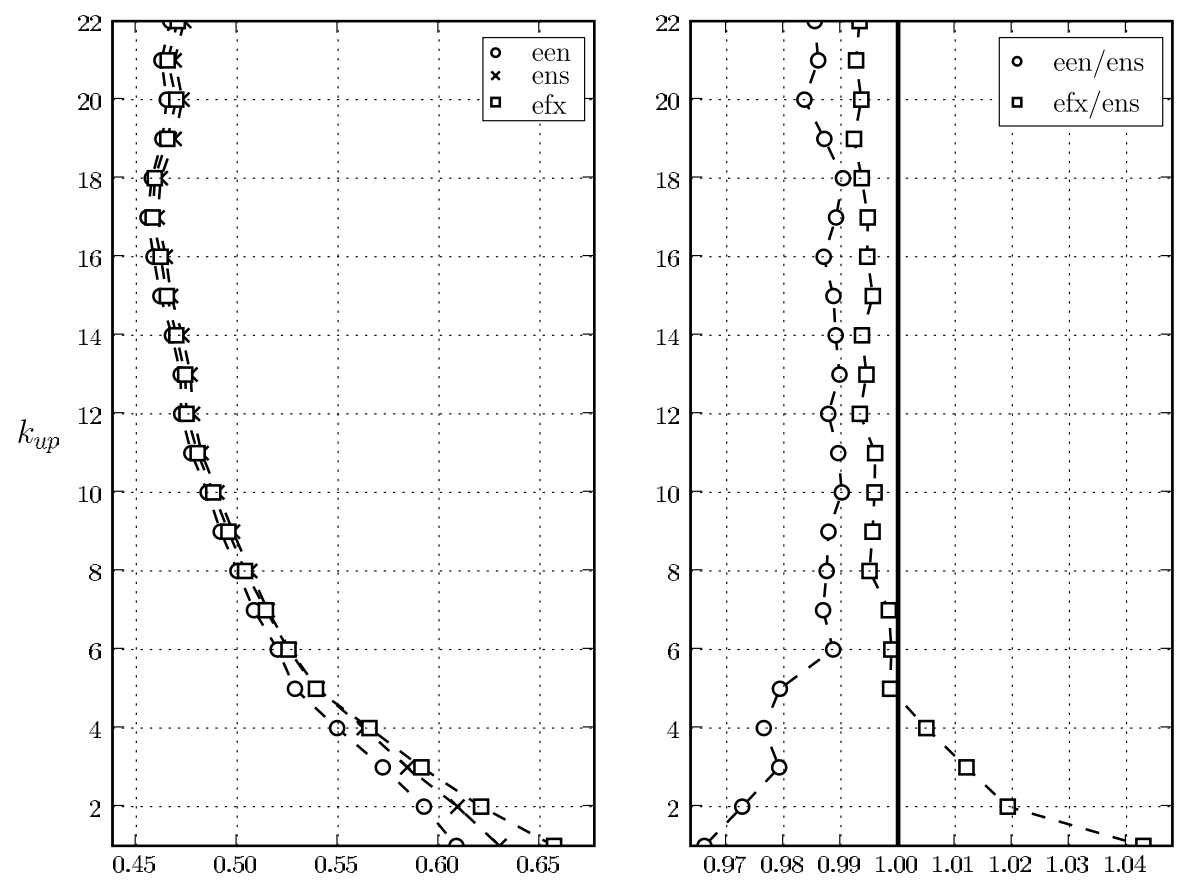

Fig. 13. Irregularity of $\mathcal{T}_{\zeta}$ the vorticity tendency due to horizontal advection as computed by schemes ens, een and ef $x$ during the summer of year 11 in run EFX. 


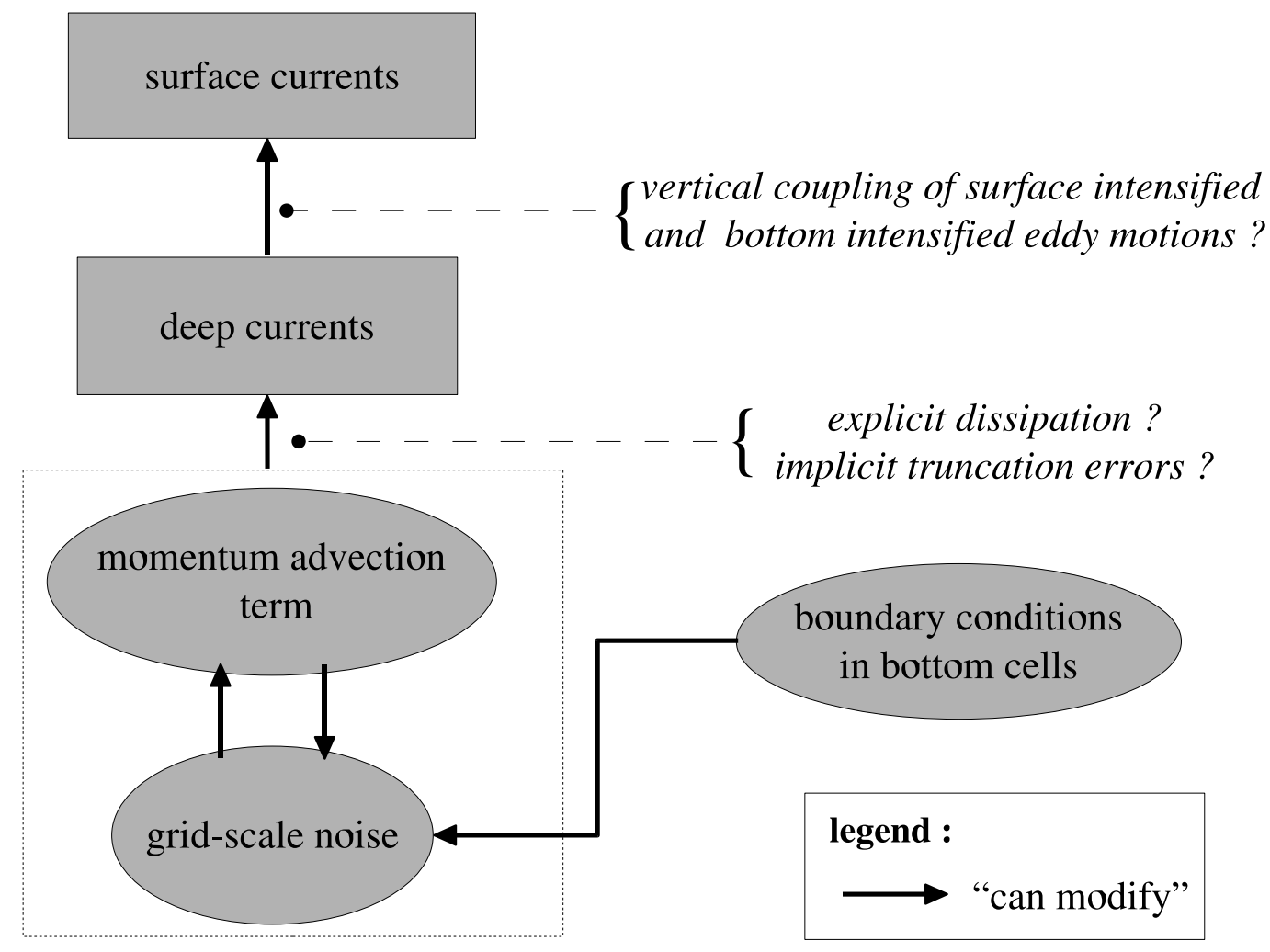

Fig. 14. Diagram synthesizing the conjectured mechanism responsible for the spreading of our three model runs. 
List of Tables 


\begin{tabular}{cccc}
\hline & $i$ & $j$ & $k$ \\
\hline$u$ & 1 & $\frac{1}{2}$ & 1 \\
$v$ & $\frac{1}{2}$ & 1 & 1 \\
$w$ & $\frac{1}{2}$ & $\frac{1}{2}$ & $\frac{1}{2}$ \\
$f, \zeta$ & 1 & 1 & 1 \\
$T, S$ & $\frac{1}{2}$ & $\frac{1}{2}$ & 1 \\
\hline
\end{tabular}

Table 1. Indices of the variables on a C-grid. An entry of 1 or $\frac{1}{2}$ indicates whether a variable is located at an integer or half value of the index. 